KCL-MTH-01-39

\title{
Superfield representations of superconformal groups
}

\author{
P.J.Heslop \\ Department of Mathematics \\ King's College, London
}

\begin{abstract}
Representations of four dimensional superconformal groups are constructed as fields on many different superspaces, including super Minkowski space, chiral superspace, harmonic superspace and analytic superspace. Any unitary irreducible representation can be given as a field on any one of these spaces if we include fields which transform under supergroups. In particular, on analytic superspaces, the fields are unconstrained. One can obtain all representations of the $N=4$ complex superconformal group $P S L(4 \mid 4)$ with integer dilation weight from copies of the Maxwell multiplet on $(4,2,2)$ analytic superspace. This construction is compared with the oscillator construction and it is shown that there is a natural correspondence between the oscillator construction of superconformal representations and those carried by superfields on analytic superspace.
\end{abstract}




\section{Introduction}

Recently there has been renewed interest in the study of unitary irreducible representations of superconformal groups in the light of the AdS/CFT correspondence [1]. This correspondence relates $\mathrm{M}$ theory or string theory on $A d S \times S$ backgrounds to superconformal field theories which can be thought of as living on the boundary of the $A d S$ space. The bulk theory and the boundary theory both have the same symmetry group, and the operators in both theories fall into representations of the superconformal group.

There are different methods of constructing these representations [2-11]. In this paper we show how to construct any unitary irreducible representation of the four dimensional superconformal groups $S U(2,2 \mid N)$ explicitly, acting on superfields on a number of different superspaces. These spaces include super Minkowski space, chiral superspaces, harmonic superspaces and analytic superspaces. Until recently [12-14] only superfields which transform trivially under semisimple supergroups have been considered. It is by allowing superfields to carry non-trivial representations of supergroups (i.e. by allowing superfields with superindices) that we are able to obtain all unitary irreducible representations as superfields. In particular, we show that all unitary irreducible representations are carried by unconstrained fields on analytic superspaces.

The techniques here may be thought of as the natural application of twistor techniques to the supersymmetric case. However things are much simpler in this case as we need not consider higher cohomology. Indeed it is only on super (ambi-) twistor spaces that one needs higher cohomology to describe superconformal representations. For all other superspaces unitary irreducible representations are carried by superfields.

A number of papers have been written in which superconformal field theories are studied on analytic superspaces [15-21]. The advantage of these spaces is that the fields are automatically irreducible and thus the full power of superconformal invariance can be exploited more easily. By allowing fields which transform non-trivially under supergroups we are able to give all unitary irreducible representations as unconstrained fields on analytic superspace. Therefore representations not previously considered in the analysis can be included, and in principle the whole theory could be given in analytic superspace (see $[13,14]$ for recent work using these techniques).

In section 2 we discuss on-shell massless multiplets and their manifestation as constrained fields on super Minkowski space. We review harmonic superspaces, first introduced by GIKOS [22-26], and how to obtain all series $\mathrm{B}$ and $\mathrm{C}$ representations as analytic fields on these, which are invariant under supergroups [27]. We then have a brief look at representations on $N=2$ analytic superspace and $(4,2,2)$ analytic superspace which transform under supergroups.

In section 3 we review various aspects of supergroup representation theory using the method of parabolic induction. We consider super Dynkin diagrams (or Kac-Dynkin diagrams), which will have crosses through to indicate the space, and numbers above the nodes to indicate the representation. We review the unitary bounds and give these as bounds on the allowed Dynkin labels.

In section 4 we discuss the finding of irreducible representations firstly in the context of Verma modules, and then translate this to Minkowski space, giving the constraints fields must satisfy 
in order to carry irreducible representations. We then apply these techniques to the supersymmetric case and give the constraints that superfields on super Minkowski space must satisfy in order to carry unitary irreducible representations. Finally we show that these correspond to unconstrained superfields on analytic superspace.

In section 5 we look at finite dimensional representations of supergroups using superindices and super Young tableaux. We show how to construct any representation on any allowed space as a tensor (or quasi-tensor) superfield. We consider examples in $N=2$ analytic superspace and show how to construct all representations (with quantised dilation weight) in $(4,2,2)$ analytic superspace from the basic Maxwell multiplet.

In section 6 we look at the oscillator method of obtaining representations. Firstly we look at representations of the conformal group and show the relationship between oscillator representations and representations as conformal fields on Minkowski space. If we apply this method for superconformal representations one is naturally lead to representations carried by superfields on analytic superspace.

There is an appendix in which we review various aspects of conformal representations as fields on Minkowski space and the irreducibility conditions they satisfy. The results of this paper come from applying these techniques in the superspace context.

\section{Superconformal fields}

\subsection{Massless multiplets on super Minkowski space}

An important class of operators consists of those which undergo shortening since they are protected from renormalisation. On super Minkowski space the shortening conditions occur as differential operators acting on the superfields. For example, on-shell massless supermultiplets can be given as constrained fields on super Minkowski space [28-30]. There are three classes of massless supermultiplets.

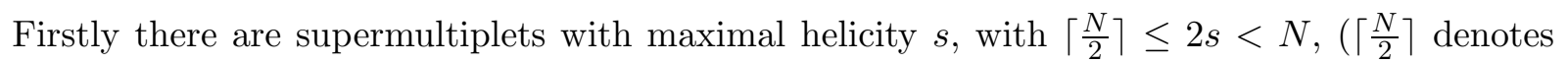
the nearest integer greater than or equal to $\frac{N}{2}$ ). These are described in (real) super Minkowski space, $M$, by superfields $W$ which have $p=2 s$ totally antisymmetric internal indices and which satisfy

$$
\begin{aligned}
\bar{D}_{\dot{\alpha}}^{i} W_{j_{1} \ldots j_{p}} & =\frac{p(-1)^{p-1}}{N-p+1} \delta_{\left[j_{1}\right.}^{i} \bar{D}_{\dot{\alpha}}^{k} W_{\left.j_{2} \ldots j_{p}\right] k} \\
D_{\alpha i} W_{j_{1} \ldots j_{p}} & =D_{\alpha[i} W_{\left.j_{1} \ldots j_{p}\right]}
\end{aligned}
$$

For each such superfield there is a conjugate superfield $\tilde{W}_{i_{1} \ldots i_{N-p}}$ defined by

$$
\tilde{W}_{i_{1} \ldots i_{N-p}}=\frac{1}{p !} \varepsilon_{i_{1} \ldots i_{N-p} j_{N-p+1} \ldots j_{N}} \bar{W}^{j_{N-p+1} \ldots j_{N}}
$$

satisfying similar constraints. When $s=\frac{1}{4} N \in \mathbb{Z}^{+}$, the multiplet is self-conjugate:

$$
\bar{W}^{i_{1} \ldots i_{p}}=\frac{1}{p !} \varepsilon^{i_{1} \ldots i_{p} j_{1} \ldots j_{p}} W_{j_{1} \ldots j_{p}}
$$


We therefore have scalar superfields $W_{i_{1} \ldots i_{p}}$ antisymmetric on $p$ internal indices with $p$ ranging from 1 to $N-1$ all of which obey the constraints (1). The second case extends this to $p=0$ and $p=N$ where we have $s=\frac{N}{2}$ and (1) implies that the field is chiral or anti-chiral respectively. These must satisfy a further second order constraint in order to be irreducible. So the chiral field $W$ satisfies

$$
\bar{D}_{\dot{\alpha}}^{i} W=0, \quad D_{\alpha i} D_{j}^{\alpha} W=0 .
$$

and the antichiral field $\bar{W}$ satisfies

$$
D_{\alpha i} \bar{W}=0, \quad \bar{D}_{\dot{\alpha}}^{i} D^{\dot{\alpha} j} \bar{W}=0 .
$$

The third case consists of massless multiplets with maximal helicity $s=J_{1}+\frac{N}{2}$ which correspond to chiral superfields with spacetime indices $W_{\alpha_{1} \cdots \alpha_{2 J_{1}}}$. These must satisfy another constraint in addition to the chiral constraint in order to form an irreducible representation of the superconformal group:

$$
\bar{D}_{\dot{\alpha}}^{i} W_{\alpha_{1} \cdots \alpha_{2 J_{1}}}=0 \quad D_{i}^{\alpha} W_{\alpha \alpha_{2} \cdots \alpha_{2 J_{1}}}=0
$$

Conjugate massless multiplets are defined similarly as antichiral fields $W_{\dot{\alpha}_{1} \cdots \dot{\alpha}_{2 J_{2}}}$ satisfying the complex conjugate constraints

$$
D_{\alpha i} W_{\dot{\alpha} \dot{\alpha}_{2} \cdots \dot{\alpha}_{2 J_{2}}}=0 \quad \bar{D}^{\dot{\alpha} i} W_{\dot{\alpha} \dot{\alpha}_{2} \cdots \dot{\alpha}_{2 J_{2}}}=0 .
$$

\subsection{Harmonic superspaces}

Harmonic superspaces are usually taken to be of the form $M \times F$ where $M$ is super Minkowski space and $F=P \backslash G$ is a coset space of the internal symmetry group $G$ with isotropy group $P[22-25,31,32]$. The harmonic superspace with $P=S(U(p) \times U(N-p-q) \times U(q))$ is known as $(N, p, q)$ harmonic superspace which we will use here. Generalised $(N, p, q)$ harmonic superspaces can be defined similarly, but with $U(N-p-q)$ replaced with some subgroup of $U(N-p-q)$.

In the GIKOS formalism [22] one instead works with fields defined on $M \times G$ which are equivariant with respect to $P$. That is, one has fields $A(z, u), z \in M, u \in G$ which take their values in some representation space of $P$ and which satisfy $A(z, h u)=R(h) A(z, u), R$ being the representation of $P$ in question. In index notation we write $u_{I}^{i}$ where the upper case index indicates a representation of $H$ and the lower case index the defining representation of $G$, and where both indices run from 1 to $N$. We can use $u$ to convert $G$ indices to $H$ indices. For $(N, p, q)$ harmonic superspaces we split the index $I$ as follows under $H: I=\left(r, r^{\prime \prime}, r^{\prime}\right)$, where $r=1, \ldots p, r^{\prime \prime}=p+1, \ldots N-q, r^{\prime}=N-q+1, \ldots N$, and where $(p+q) \leq N$, then we can have superfields $A$ which obey the constraints

$$
D_{\alpha r} A=\bar{D}_{\dot{\alpha}}^{r^{\prime}} A=0
$$

where

$$
D_{\alpha r}:=u_{r}^{i} D_{\alpha i} ; \quad \bar{D}_{\dot{\alpha}}^{r^{\prime}}=\bar{D}_{\dot{\alpha}}^{i}\left(u^{-1}\right)_{i}^{r^{\prime}}
$$

Constraints such as these are called Grassmann analyticity (G-analyticity) conditions following [23]. The derivatives on $G$ can be taken to be the right-invariant vector fields, $D_{I}^{J}, D_{I}^{I}=0$. They act as follows:

$$
D_{I}^{J} u_{K}^{k}=\delta_{K}^{J} u_{I}^{k}-\frac{1}{N} \delta_{I}^{J} u_{K}^{k}
$$


and they obey the reality condition

$$
\bar{D}^{J}{ }_{I}=-D_{I}^{J} .
$$

They divide into three sets; the set $\left\{D_{r} s^{\prime}, D_{r} s^{\prime \prime}, D_{r^{\prime \prime}} s^{\prime}\right\}$, corresponding to the $\bar{\partial}$ operator on the complex coset space (i.e. holomorphic functions are annihilated by $\bar{\partial}$ ), the complex conjugate set $\left\{D_{r^{\prime}}{ }^{s}, D_{r^{\prime \prime}} s, D_{r^{\prime}} s^{\prime \prime}\right\}$ which corresponds to the $\partial$ operator, and the set $\left\{D_{r} s, D_{r^{\prime}} s^{\prime}, D_{r^{\prime \prime}} s^{\prime \prime}\right\}$ which corresponds to the isotropy algebra. As well as imposing G-analyticity we can impose harmonic analyticity (H-analyticity) by demanding that a superfield be annihilated by the first of these sets of derivatives. Indeed, since the algebra of all of these operators and the spinorial derivatives defining G-analyticity closes, it is consistent to impose both G-analyticity and H-analyticity on the same superfield. Such superfields will be referred to as CR-analytic, or simply analytic.

Many of the massless multiplet fields defined above are described naturally as analytic fields on harmonic superspace. The superfields $W_{i_{i} \ldots i_{p}}$, satisfying (1), can be naturally described as CR-analytic fields on $(N, p, N-p)$ harmonic superspaces [32]. Define the scalar superfield

$$
W=\frac{1}{p !} \varepsilon^{r_{1} \cdots r_{p}} u_{r_{1}}^{i_{1}} \cdots u_{r_{p}}^{i_{p}} W_{i_{1} \cdots i_{p}}
$$

on $(N, p, q)$ harmonic superspace. Then the conditions (1) become G-analyticity constraints on $W$

$$
\begin{aligned}
D_{\alpha r} W^{(p)} & =0 \\
\bar{D}_{\dot{\alpha}}^{r^{\prime}} W^{(p)} & =0
\end{aligned}
$$

and the H-analyticity constraint,

$$
D_{r}{ }^{\prime} W^{(p)}=0
$$

and we see that $W$ is equivariant with respect to the isotropy group $S(U(p) \times U(N-p)$. Conversely, an equivariant field with the same charge (under the $U(1)$ subgroup of the isotropy group) as $W$ and satisfying the above analyticity constraints is equivalent to a superfield on Minkowski superspace satisfying (1). We can apply this construction to the entire family of scalar superfields with $p$ varying from 0 to $N$, bearing in mind that the chiral fields satisfy the additional constraint (4).

Whilst $(N, p, N-p)$ harmonic superspace is perhaps the most natural harmonic superspace on which to represent these massless multiplets, there are many other ways of representing them that are "less efficient" in that the superspaces have more odd coordinates [27]. For example, any of the above massless multiplets, $W^{(p)}$, (except $\left.p=0, N\right)$ can be realised with $(1,1)$ G-analyticity on an internal space $F=U(1)^{N-1} \backslash S U(N)$. We define

$$
W_{1 \ldots p}=u_{1}^{i_{1}} \ldots u_{p}^{i_{p}} W_{i_{1} \ldots i_{p}}
$$

where $W_{i_{1} \ldots i_{p}}$ is the superfield on super Minkowski space. Equation (1) is equivalent to the G-analyticity conditions

$$
\begin{aligned}
D_{\alpha 1} W_{1 \ldots p} & =0 \\
\bar{D}_{\dot{\alpha}}^{N} W_{1 \ldots p} & =0
\end{aligned}
$$

and the H-analyticity conditions

$$
D_{I}{ }^{J} W=0 \text { for } I<J \text {. }
$$


Unitary representations of the superconformal group fall into three series which have been called A, B and C (see section 3.4). Series B and C correspond to short multiplets. There is a very simple way of generating all possible series $\mathrm{B}$ and $\mathrm{C}$ representations from the set of available massless supermultiplets for a given $N$ using this harmonic superspace $[27,33]$. Firstly, for series $\mathrm{C}$, if $\left(a_{1}, \ldots, a_{N-1}\right)$ are the internal group Dynkin labels of the representation we are interested in, simply form the product

$$
A=\prod_{k=1}^{N-1}\left(W_{12 \ldots k}\right)^{a_{k}} .
$$

The dilation weight is equal to the sum of the Dynkin labels, $m_{1}=\sum_{k=1}^{N-1} a_{k}$, since each underlying field has dilation weight 1 . Furthermore the field $W_{12 \ldots k}$ has R-charge $\frac{2 k}{N-1}$ and so the field $A$ will have R-charge $\frac{2 m}{N-m_{1}}$ where $m=\sum_{k=1}^{N-1} k a_{k}$.

For series B representations we work on $(N, 0,1)$ superspace, again with the internal manifold $F=U(1)^{N-1} \backslash S U(N)$. We use the same family of superfields as before augmented with the chiral fields from the previous section, $W, W_{\alpha_{1}, \ldots, \alpha_{2 J_{1}}}$. On this harmonic superspace these satisfy both analytic and non-analytic constraints (from $(4,6)$ ):

$$
\begin{aligned}
& \bar{D}_{\dot{\alpha}}^{N} W=0 \quad D_{\alpha 1} D_{1}^{\alpha} W=0 \\
& \bar{D}_{\dot{\alpha}}^{N} W_{\alpha_{1} \ldots \alpha_{2 J_{1}}}=0 \quad D_{1}^{\alpha} W_{\alpha \alpha_{2} \ldots \alpha_{2 J_{1}}}=0 \text {. }
\end{aligned}
$$

We can then form the product superfield $[27,33]$

$$
A_{\alpha_{1} \ldots \alpha_{2 J_{1}}}=W_{\alpha_{1} \ldots \alpha_{2 J_{1}}} W^{b} \prod_{k=1}^{N-1}\left(W_{12 \ldots k}\right)^{a_{k}}
$$

where $b$ is a positive number (we allow non-integer powers to obtain representations with noninteger dilation weight). The $L$ weight of this field is $L=m_{1}+b+J_{1}$ and the R-symmetry weight is $R=2 m / N-\left(m_{1}+b+J_{1}+1\right)$ and therefore $L+R=2 m / N$ as required (see (69)). In the case $b=0$ these fields will satisfy the additional constraints $D_{1}^{\alpha} A_{\alpha \alpha_{2} \ldots \alpha_{2 J_{1}}}=0$ if $J_{1}$ is non-zero, or $D_{\alpha 1} D_{1}^{\alpha} A=0$ if $J_{1}$ is zero. These representations saturate the bounds of (69).

We can also produce the series A fields if we also include antichiral fields $\bar{W}$ and $\bar{W}_{\dot{\alpha}_{1}, \ldots, \dot{\alpha}_{J_{2}}}$. We form the field [33]

$$
W_{\alpha_{1} \ldots \alpha_{2 J_{1}}} \bar{W}_{\dot{\alpha}_{1} \ldots \dot{\alpha}_{J_{2}}} W^{b} \bar{W}^{c} \prod_{k=1}^{N-1}\left(W_{12 \ldots k}\right)^{a_{k}}
$$

where $b, c \geq 0$. This field is not an analytic superfield and in general is unconstrained although in the case $b=0$ or $c=0$ we obtain further constraints.

\subsection{All representations as analytic fields}

In this paper we give a systematic study of unitary superconformal representations as superfields on different superspaces with the aid of super Dynkin diagrams. In fact all unitary irreducible representations of the superconformal group can be given as superfields on many different superspaces, and in particular on analytic superspaces all fields carry irreducible representations. We complexify spacetime and the superconformal group to $S L(4 \mid N)$. Then analytic superspaces 
can be exhibited as coset spaces even though they are not coset spaces of the real superconformal group $S U(2,2 \mid N)$. On complex $(N, p, q)$ superspace fields transform under the group $S(G L(2 \mid p) \times G L(N-p-q) \times G L(2 \mid q))$. Only fields which are invariant under the supergroups $S L(2 \mid p)$ and $S L(2 \mid q)$ have been considered until recently [12-14]. If we relax this and allow fields that transform under supergroups then all representations can be given as fields on any superflag space (except super (ambi-) twistor spaces for technical reasons we will explain later.)

For example, consider $N=2$ analytic superspace. Fields on this space transform under $S(G L(2 \mid 1) \times G L(2 \mid 1)) \backslash S L(4 \mid 2)$. The coordinates of analytic superspace are

$$
X^{A A^{\prime}}=\left(\begin{array}{cc}
x^{\alpha \dot{\alpha}} & \lambda^{\alpha} \\
\pi^{\dot{\alpha}} & y
\end{array}\right)
$$

where $x$ are the usual (complex) spacetime coordinates, $\lambda, \pi$ are odd coordinates, and $y$ is the coordinate of the internal manifold $\mathbb{C} P^{1}$. We have already seen (in the real case) that the hypermultiplet can be exhibited as an analytic field. The hypermultiplet is an $N=2$ massless multiplet with highest spin $s=\frac{1}{2}$ and so it is given on super Minkowski space by a field $W_{i}$ satisfying equations (1) and on harmonic superspace by a field $W$ satisfying the analytic constraints $(13,14)$. In the complex setting this is an unconstrained field on analytic superspace invariant under both $S L(2 \mid 1)$ supergroups but transforming with a $\mathbb{C}$-weight.

A more unusual multiplet to consider as an analytic field is the $N=2$ Maxwell multiplet. This is normally given as a chiral field on super Minkowski space, satisfying the additional constraint (4). On analytic superspace it is given as a holomorphic field $W_{A}$ transforming contravariantly under the first $S L(2 \mid 1)$ subgroup, and satisfying no constraints. The conjugate multiplet is given similarly by a field $W_{A^{\prime}}$ transforming under the second $S L(2 \mid 1)$ subgroup.

Another example is the $N=2$ superconformal stress-energy tensor, which on super Minkowski space is a scalar superfield satisfying a second order constraint. On analytic superspace it is given by a field $T_{A^{\prime} A}$ transforming under both $S L(2 \mid 2)$ supergroups. Once more this irreducible representation is given as an unconstrained field on analytic superspace. This representation can also be realised by multiplying massless multiplets together in two different ways on analytic superspace: firstly by multiplying a Maxwell field and its conjugate together

$$
T_{A^{\prime} A}=W_{A^{\prime}} W_{A}
$$

and secondly by multiplying two hypermultiplet fields together with a derivative:

$$
T_{A^{\prime} A}=W_{1} \partial_{A^{\prime} A} W_{2}-W_{2} \partial_{A^{\prime} A} W_{1} .
$$

In the case $N=4$ consider $(4,2,2)$ analytic superspace. The coordinates of $(4,2,2)$ analytic superspace are given as

$$
X^{A A^{\prime}}=\left(\begin{array}{cc}
x^{\alpha \dot{\alpha}} & \lambda^{\alpha a^{\prime}} \\
\pi^{a \dot{\alpha}} & y^{a a^{\prime}}
\end{array}\right)
$$

where again $x$ are spacetime coordinates, $\lambda, \pi$ are odd coordinates and $y$ are coordinates for the internal manifold. The group $S L(4 \mid 4)$ is not semisimple and thus one normally divides out the centre to leave $P S L(4 \mid 4)$. For representations this simply means only those with vanishing 
R-charge are allowed. For fields on analytic superspace the condition $R=0$ turns out to be the condition that the number of primed indices must equal the number of unprimed indices. The only massless multiplet with vanishing $\mathrm{R}$-charge is the $N=4$ Maxwell multiplet. Given on super Minkowski space as the field $W_{i j}$ it becomes the scalar field $W$ on analytic superspace, defined as in (12). As we will argue later one can in fact produce all representations of the supergroup PSL(4|4) using copies of the Maxwell multiplet, together with derivatives. For example the field

$$
W_{A^{\prime} A}=W^{(1)} \partial_{A^{\prime} A} W^{(2)}-W^{(2)} \partial_{A^{\prime} A} W^{(1)}
$$

where $W^{(i)}$ are different copies of the Maxwell multiplet on $(4,2,2)$ analytic superspace, corresponds to the series $\mathrm{C}$ representation with internal Dynkin labels $[1,0,1]$ and dilation weight $L=2$.

\section{Representations of supergroups}

We wish to apply the techniques outlined in the appendix for Lie groups to simple Lie supergroups, concentrating in particular on the complex group $S L(r \mid s)$ and sometimes specifically the complex superconformal group $S L(4 \mid N)$. Many concepts can be carried over from the purely bosonic case with some important differences which we will point out when they occur. See [34] for a review of super Lie groups and their representations.

\subsection{Roots and parabolic subalgebras of $S L(r \mid s)$}

We set the Cartan subalgebra $\mathfrak{h}$ of $\mathfrak{s l}(r \mid s)$ to be the set of supertraceless diagonal matrices. The roots are given by $e_{i j} \in \mathfrak{h}^{*}$ with $1 \leq i \neq j \leq r+s$ where

$$
e_{i j}(h)=h_{i}-h_{j} \quad \mathfrak{h} \ni h=\operatorname{diag}\left(h_{1}, h_{2}, \ldots, h_{r} \mid h_{r+1}, h_{r+2}, \ldots, h_{r+s}\right)
$$

and where the $h_{i}$ are any Grassmann even numbers, subject to the constraint

$$
\sum_{k=1}^{r} h_{k}-\sum_{k=r+1}^{r+s} h_{k}=0 .
$$

The subspaces of $\mathfrak{s l}(r \mid s), \mathfrak{g}_{e_{i j}}$ corresponding to these roots are spanned by the matrices $\hat{e}_{j i}$ which have zeros everywhere except in the $j i$ component where there is a 1 . A root is said to be even or odd according to whether the non zero component of $\mathfrak{g}_{e_{i j}}$ is even or odd. We now choose a system of simple roots, for example for $S L(4 \mid N)$ the set of roots

$$
S_{D}=\left\{e_{i(i+1)}: i=1 \ldots 3+N\right\} .
$$

This particular choice of roots is called the distinguished system of simple roots as it has only one odd root $e_{45}$. However unlike in the purely bosonic case, where all choices of simple roots are equivalent up to conjugation, we can now have inequivalent systems of simple roots. For example, another choice for a system of simple roots for $S L(4 \mid N)$, which will turn out to be the one we will use, is

$$
S=\left\{e_{12}, e_{25}, e_{67}, \ldots, e_{(3+N)(4+N)}, e_{(4+N) 3}, e_{34}\right\} .
$$


This has two odd roots $e_{25}$ and $e_{(4+N) 3}$. The easiest way to see that these are inequivalent is by constructing the respective standard Borel subalgebras from them.

Given a choice of simple roots we define the set of positive roots, $\Delta^{+}$, in the usual way, as in $(224)$ and then the standard Borel subalgebra $\mathfrak{b}$ is defined as in $(236,237)$. We see that the standard Borel subalgebra for $S L(4 \mid N)$ constructed with the choice of simple roots $S_{D}$, is simply the set of lower triangular matrices

$$
\mathfrak{b}_{S_{D}}=\left\{\left(\begin{array}{cccc|ccc}
\bullet & & & & & \\
\bullet & \bullet & & & & \\
\bullet & \bullet & \bullet & & & \\
\bullet & \bullet & \bullet & \bullet & & \\
\hline \bullet & \bullet & \bullet & \bullet & \bullet & & \\
\bullet & \bullet & \bullet & \bullet & \cdot & \cdot & \\
\bullet & \bullet & \bullet & \bullet & \bullet & \cdot & \bullet
\end{array}\right)\right\}
$$

whereas the Borel subalgebra constructed using $S$ is given by matrices with the following form:

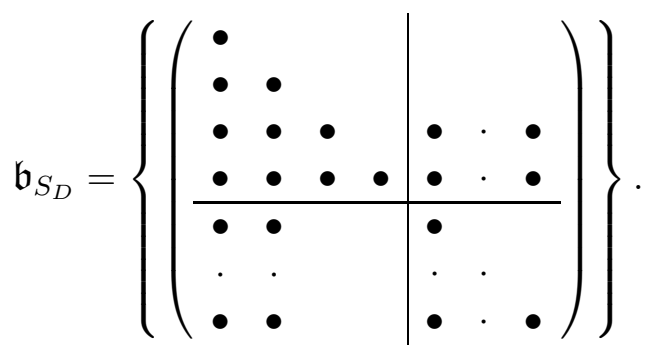

However, if we make a simple change of basis for $\mathbb{C}^{4 \mid N}$ on which the $S L(4 \mid N)$ matrix acts, we can change an element in the Lie algebra $\mathfrak{s l}(4 \mid N)$ as follows:

$$
g=\left(\begin{array}{l|l}
4 & \\
& \\
\hline & N
\end{array}\right) \rightarrow\left(\begin{array}{l|l|l}
2 & & \\
\hline & N & \\
\hline & & 2
\end{array}\right) .
$$

In this new basis the Borel subalgebra becomes lower triangular again

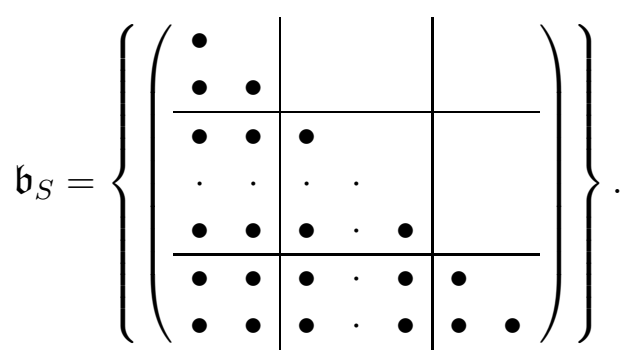

For the supergroup $S L(r \mid s)$, for any choice of simple roots, we can always choose a basis for $\mathbb{C}^{r \mid s}$ on which the group acts such that the Borel subgroup is the set of lower triangular matrices. We see from our example that this change of basis can not be performed using an $S L(r \mid s)$ matrix as it swaps bosonic and fermionic components around. So $\mathfrak{b}_{S}$ is not equivalent to $\mathfrak{b}_{S_{D}}$ under conjugation (i.e. $\mathfrak{b}_{S} \neq g \mathfrak{b}_{S_{D}} g^{-1}$ for any $g \in G$ ) and the choices of simple roots are inequivalent. 
The Killing form for a superalgebra $\mathfrak{g}$ is defined as

$$
(u, v)=\operatorname{str} \operatorname{ad}(u) \operatorname{ad}(v)
$$

for $u, v \in \mathfrak{g}$, where ad denotes the adjoint representation. This simplifies in the case $S L(r \mid s)$ to

$$
(u, v)=2(r-s) \operatorname{str} u v \quad r \neq s .
$$

In the case $r=s$ this vanishes but a convenient non-degenerate bilinear form that can be used in its place is provided by [34]

$$
(u, v)=\operatorname{str} u v
$$

and we shall mean this, when $r=s$, when we refer to the Killing form. We use this Killing form to identify $\mathfrak{h}$ with $\mathfrak{h}^{*}$ just as in the purely bosonic case (226). However $(\alpha, \alpha)$ is no longer necessarily positive for any root $\alpha$. Indeed, if $\alpha$ is an odd root it is possible that $(\alpha, \alpha)=0$. We define the co-root $\alpha^{\vee}$ of a simple root $\alpha$ as

$$
\alpha^{\vee}=\left\{\begin{array}{cc}
2 \alpha /(\alpha, \alpha) & \text { if }(\alpha, \alpha) \neq 0 \\
\alpha /(\alpha, \beta) & \text { if }(\alpha, \alpha)=0
\end{array}\right.
$$

where we choose a simple root $\beta \in S$ such that $(\alpha, \beta) \neq 0$. The Cartan matrix is defined just as in (230). The Dynkin diagram is defined from the Cartan matrix in exactly the same way as in the bosonic case, except we now distinguish between even and odd simple roots by assigning black and white nodes to them respectively. The Dynkin diagram for $\mathfrak{g}$ is not unique and depends on the choice of simple roots, inequivalent systems of simple roots giving rise to different Dynkin diagrams.

The Dynkin diagram for $S L(4 \mid N)$ obtained from the distinguished system of simple roots $S_{D}$ is

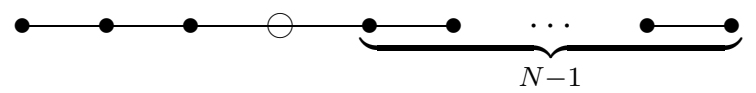

Here the three nodes on the left correspond to the $\mathfrak{s l}(4)$ subgroup of $\mathfrak{s l}(4 \mid N)$, the $N-1$ nodes on the right correspond to the $\mathfrak{s l}(N)$ subgroup of $\mathfrak{s l}(4 \mid N)$, and the white node corresponds to the odd root. The Dynkin diagram obtained using the system of simple roots $S$ is

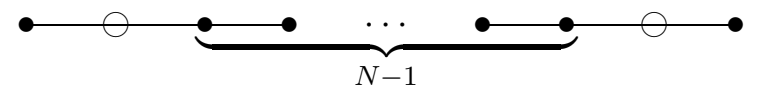

Parabolic subalgebras are ones which contain the Borel subalgebra, and they can be constructed in the same way as in the bosonic case, by specifying a subset $S_{\mathfrak{p}}$ of the simple roots $S$ and finding $\mathfrak{l}$ and $\mathfrak{n}$ (see (238-241). For $\mathfrak{s l}(r \mid s)$, once we have obtained a basis for $\mathbb{C}^{r \mid s}$ with respect to which the Borel subalgebra consists of lower triangular matrices, the parabolic subalgebras are simply the sets of block lower triangular matrices of a particular shape, and the corresponding Levi subalgebras are the block diagonal parts. They can be represented on the Dynkin diagram for $\mathfrak{s l}(r \mid s)$ by crossing out the nodes corresponding to simple roots in $S \backslash S_{\mathfrak{p}}$.

All compactified complexified superspaces that have been used in the study of four dimensional flat space supersymmetric field theories are of the form $P \backslash S L(4 \mid N)$ where $P$ is a parabolic subgroup represented by putting crosses on the Dynkin diagram (41). 
For example, compactified complexified super Minkowski space $\tilde{\mathbb{M}}$ has the form $P \backslash S L(4 \mid N)$ where $P$ consists of matrices of the form

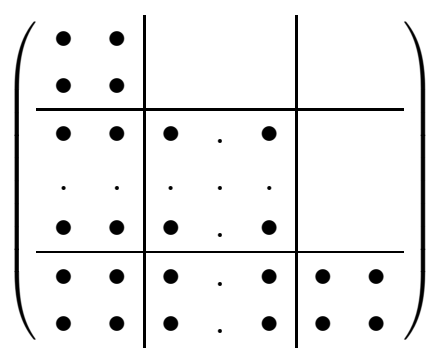

with unit superdeterminant. The corresponding Levi subgroup $L$ is the set of block diagonal matrices of the form

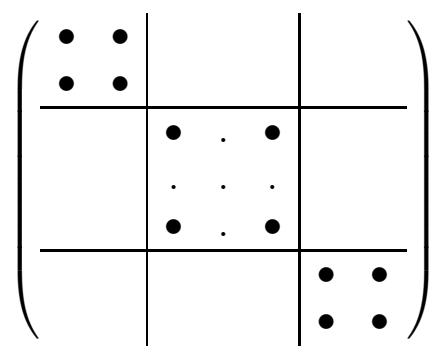

with unit superdeterminant. This parabolic subalgebra can be constructed using the choice of simple roots $S$ in (31) and with $S_{\mathfrak{p}}=\left\{e_{25}, e_{(4+N) 3}\right\}$ consisting of the two odd roots. It has corresponding Dynkin diagram

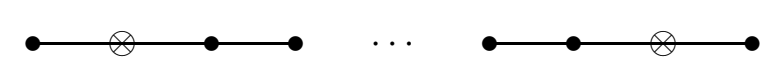

where the two odd roots have crosses through them. Note that it is very simple to read off the form of the Levi subalgebra $\mathfrak{l}$ from the Dynkin diagram. The two crossed through nodes represent $\mathbb{C}$-charges and the remaining nodes give us the form of the semisimple part $\mathfrak{l}_{S}$. Here we obtain $\mathfrak{l}=\mathfrak{s l}(2) \oplus \mathfrak{s l}(N) \oplus \mathfrak{s l}(2) \oplus \mathbb{C}^{2}$.

We can identify non-compact complex super Minkowski space $\mathbb{M}$ as an open set in compact complex super Minkowski space. The standard coset representative is

$$
M \ni z \mapsto s(z)=\left(\begin{array}{c|c|c}
1_{2} & \theta & x \\
\hline 0 & 1_{N} & \varphi \\
\hline 0 & 0 & 1_{2}
\end{array}\right) .
$$

Here we see the usual coordinates for complex super Minkowski space, with $\varphi$ denoting the $N$ dotted two-component spinorial coordinates which become the complex conjugates of the $\theta$ 's in the real case. We can think of the coordinates for a space as fitting into the blank spaces of the isotropy group as illustrated in the above case.

If $P_{1} \subset P_{2}$ are two parabolic subgroups of a group $G$, then the coset space $P_{1} \backslash G$ is a fibre bundle over $P_{2} \backslash G$ with typical fibre $P_{1} \backslash P_{2}$

$$
P_{1} \backslash G \longrightarrow P_{2} \backslash G
$$

Non-compact superspaces are open subsets of the coset spaces $P \backslash S L(4 \mid N)$ which are obtained by considering fibrations (sometimes double fibrations) starting with non-compact super Minkowski space. 
For example, left chiral superspace $\tilde{\mathbb{M}}_{L}$ has isotropy group of the form

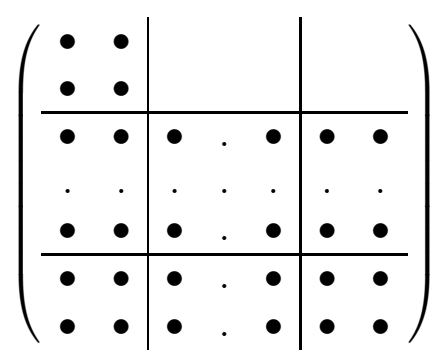

and has coordinates $(x, \theta)$. The corresponding Dynkin diagram is

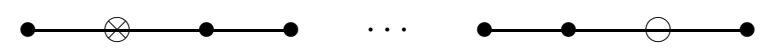

There is a fibration between compactified super Minkowski space and chiral superspace of the form (46)

$$
\tilde{\mathbb{M}} \longrightarrow \tilde{\mathbb{M}}_{L}
$$

and one defines non-compact left chiral superspace in terms of this fibration as $\pi(\mathbb{M})$. Right chiral superspace is defined similarly and has a single cross through the other odd node of the Dynkin diagram.

The spaces we will be concentrating on, however, will be harmonic superspaces. Complexified $(N, p, q)$ harmonic superspace $\tilde{\mathbb{M}}_{H}$ has the following isotropy group

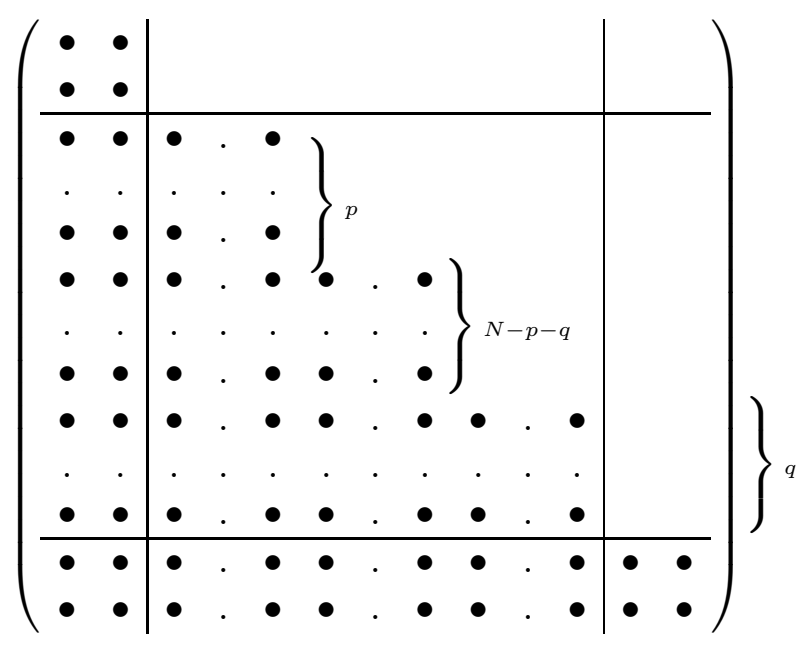

The corresponding Dynkin diagram is

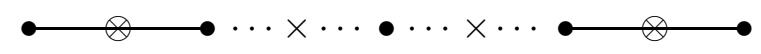

where the middle crosses are on the $p$ th and $(N-q)$ th central nodes. Again one has a fibration this time with Minkowski space as the base manifold

$$
\tilde{\mathbb{M}}_{H} \longrightarrow \tilde{\mathbb{M}}
$$


and so in the non-compact version we define $\mathbb{M}_{H}=\pi^{-1}(\mathbb{M})$ which has the form of complex super Minkowski space times an internal flag space.

The related $(N, p, q)$ analytic superspace has the same body but fewer odd coordinates. It has the following isotropy group

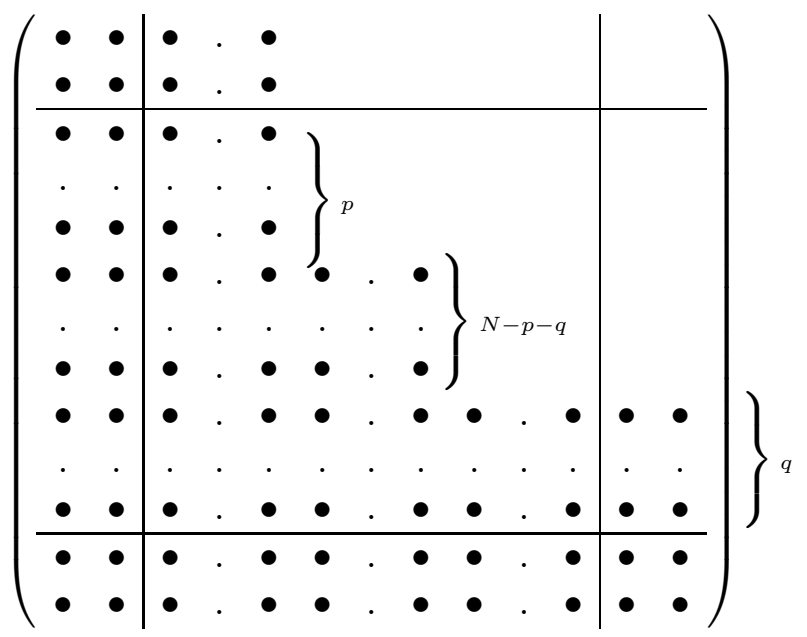

with corresponding Dynkin diagram

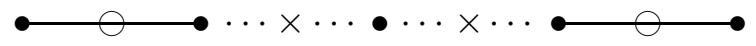

This space has only $(N-p) \theta$ 's and $(N-q) \varphi$ 's. There is a double fibration involving compactified super Minkowski space, harmonic superspace and analytic superspace

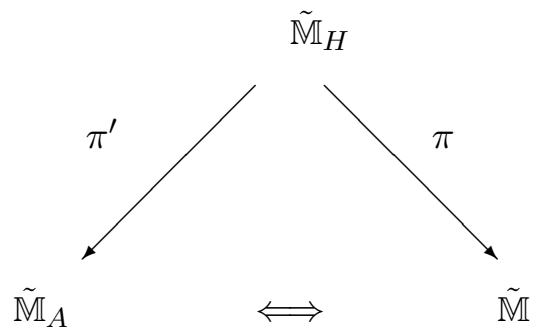

and non-compact analytic superspace is defined as $\mathbb{M}_{A}=\pi^{\prime}\left(\mathbb{M}_{H}\right)$. Generalised $(N, p, q)$ spaces can be defined, which have the same number of $\theta$ 's and $\varphi$ 's as $(N, p, q)$ space, but have a different internal space. These are given by the same Dynkin diagram as above, but with any number of extra crosses inserted between the two already there.

We note here also that the supertwistor space introduced by Ferber [35] corresponds to the Dynkin diagram

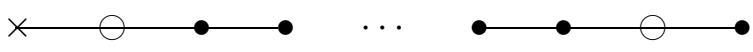




\subsection{Superconformal representations}

Weights, raising and lowering operators and highest weights are defined as in the bosonic case. Weights $\lambda_{i}$ dual to the simple roots are defined as in (247) so that any weight can be expressed uniquely as

$$
\lambda=\sum_{i}\left(\lambda, \alpha_{i}^{\vee}\right) \lambda_{i}
$$

A weight is then represented on a Dynkin diagram by putting the label $n_{j}=\left(\lambda, \alpha_{j}^{\vee}\right)$ over the $j$-th node.

For the Lie superalgebras $\mathfrak{s l}(r \mid s)$ (for $r>s \geq 1$ ) the representations with highest weight $\lambda$ are finite dimensional if and only if the even distinguished Dynkin coefficients $n_{i}^{D}$ are positive integers [34]. This is the equivalent of the dominant integral condition in the bosonic case, the difference now being that the odd Dynkin coefficient can be any (complex) number. This is important for representing operators with non-integral dilation weight as superfields on analytic superspaces.

Irreducible highest weight representations of $S L(4 \mid N)$ can therefore be defined by giving $3+N$ Dynkin coefficients. Unitary irreducible highest weight representations of the superconformal group are usually defined by giving the following $3+N$ quantum numbers: Lorentz spin, $J_{1}, J_{2}$, dilation weight, $L$, R-charge $R$, and the Dynkin labels of the internal group, $a_{1} \ldots a_{N-1}$. These can be related to the Dynkin coefficients. In order to work out this relation, we first calculate $H_{i} \in \mathfrak{h}$ dual to the simple co-roots $\alpha_{i}^{\vee} \in \mathfrak{h}^{*}$. For the choice of simple roots $S$ (31) using the new basis (34) we find that

$$
\begin{aligned}
H_{1} & =\operatorname{diag}(1,-1|0, \ldots 0| 0,0) \\
H_{2} & =\operatorname{diag}(0,-1|-1,0, \ldots, 0| 0,0) \\
H_{3} & =\operatorname{diag}(0,0|1,-1,0, \ldots, 0| 0,0) \\
\vdots & \\
H_{N+1} & =\operatorname{diag}(0,0|0, \ldots, 1,-1| 0,0) \\
H_{N+2} & =\operatorname{diag}(0,0|0, \ldots, 1| 1,0) \\
H_{N+3} & =\operatorname{diag}(0,0|0, \ldots, 0| 1,-1) .
\end{aligned}
$$

For a representation with highest weight $\lambda$, the Dynkin coefficients corresponding to the simple root $\alpha_{i}$ are given by $n_{i}=\left(\lambda, \alpha_{i}^{\vee}\right)=\lambda\left(H_{i}\right)$. The quantum numbers $Q$ given above satisfy a similar equation $Q=\lambda(\hat{Q})$ where $\hat{Q} \in \mathfrak{h}$ are as follows
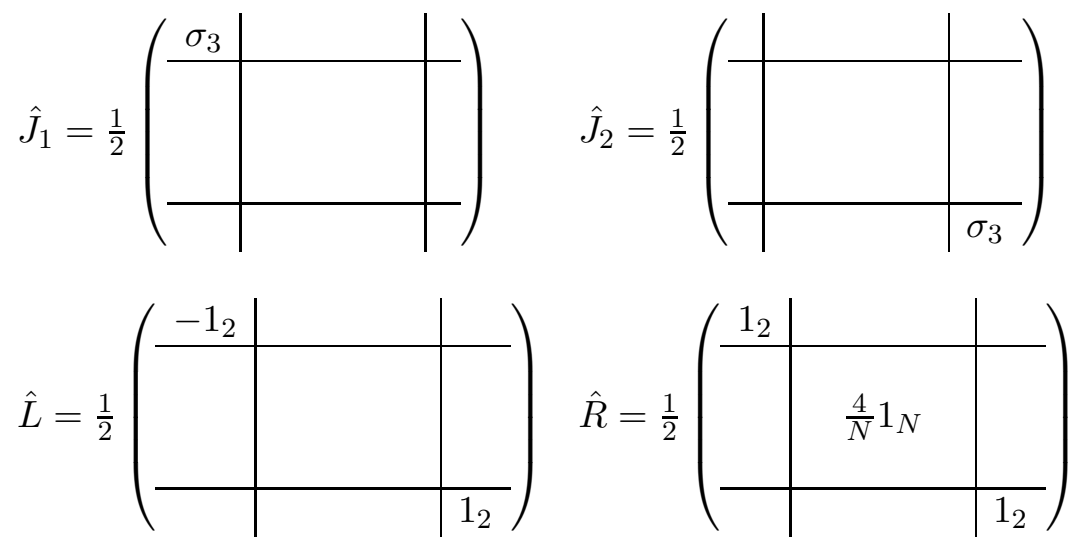
and

$$
\hat{a}_{i}=H_{i+2} \quad i=1 \ldots N-1 .
$$

We thus find that

$$
\begin{array}{lll}
n_{1}=2 J_{1} ; & n_{2}=\frac{1}{2}(L-R)+J_{1}+\frac{m}{N}-m_{1} ; & n_{2+i}=a_{i} \quad(i=1 \ldots N-1) ; \\
n_{N+3}=2 J_{2} ; & n_{N+2}=\frac{1}{2}(L+R)+J_{2}-\frac{m}{N} ; &
\end{array}
$$

where

$$
m:=\sum_{k=1}^{N-1} k a_{k} ; \quad m_{1}:=\sum_{k=1}^{N-1} a_{k} .
$$

Note that $m$ is the total number of boxes in the $\mathfrak{s l}(N)$ Young tableau, while $m_{1}$ is the number of boxes in the first row.

Finite dimensional representations of parabolic subgroups can be represented on a Dynkin diagram with crosses through it. As in the bosonic case an irreducible representation of $\mathfrak{p}=\mathfrak{l} \oplus \mathfrak{n}$ corresponds to an irreducible representation of $\mathfrak{l}$ as $\mathfrak{n}$ acts trivially. $\mathfrak{l}$ splits into a semisimple part $\mathfrak{l}_{S}$ and its centre $\mathfrak{l}_{Z}$ and thus a representation of $\mathfrak{p}$ can be specified by giving the highest weight of the representation of $\mathfrak{l}_{S}$ together with some numbers giving $\mathbb{C}$-weights (the representation of $\mathfrak{l}_{Z}$ ). Roughly speaking, on the Dynkin diagram for $\mathfrak{p}$ the Dynkin coefficients above crossed nodes correspond to the $\mathbb{C}$-weights and the remaining coefficients give the representation of $\mathfrak{l}_{S}$.

\subsection{Induced representations of supergroups}

Proceeding exactly as in the bosonic case we now describe representations of a complex simple Lie supergroup $G$ as fields on supercoset spaces $P \backslash G$ which carry a representation of the parabolic subgroup $P$. We represent these by using the Dynkin diagram for the representation of $P$ which the fields carry. For example the Wess-Zumino multiplet has quantum numbers $L=1, R=-1$, $J_{1}=J_{2}=0$ and thus, from (61), has Dynkin diagram

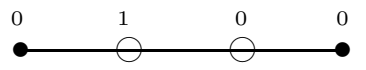

It can be represented as a chiral field (i.e. a field $A$ satisfying the constraint $\bar{D}_{\alpha} A=0$ ) on (real) $N=1$ super Minkowski space. In complex superspace this field is most naturally given as an unconstrained scalar field on $N=1$ chiral superspace (48). Its Dynkin diagram is

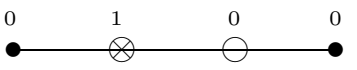

The fact that there are no non-zero coefficients above uncrossed nodes tells us that this is a scalar superfield.

The $N=2$ hypermultiplet has quantum numbers $L=1, R=0, J_{1}=J_{2}=0, a_{1}=1$ and thus has Dynkin diagram

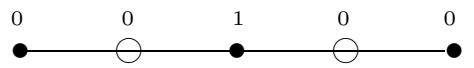

It can be given as a field on (real) super Minkowski space, $W_{i}$, carrying an $S U(2)$ index, and satisfying the constraint (1)

$$
\begin{aligned}
\bar{D}_{\dot{\alpha}}^{i} W_{j} & =\frac{1}{2} \delta_{j}^{i} \bar{D}_{\dot{\alpha}}^{k} W_{k} \\
D_{\alpha i} W_{j} & =D_{\alpha[i} W_{j]} .
\end{aligned}
$$


This has Dynkin diagram

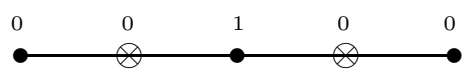

Note that this has a 1 above the central node, confirming that the field has a single $S U(2)$ index. Another alternative description of this multiplet is as a field on analytic space. Here it is an unconstrained field $W$ without indices, and has Dynkin diagram

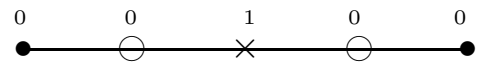

The above examples are well known and all transform trivially under supergroups. To include all UIRs on a particular space it is necessary to consider fields which transform non-trivially under supergroups.

\subsection{Unitary bounds}

Unitary irreducible highest weight representations of the superconformal group satisfy the following unitary bounds $[2-6]$ :

$$
\begin{array}{lll}
\text { A) } L \geq 2+2 J_{2}-R+\frac{2 m}{N}, & L \geq 2+2 J_{1}+R+2 m_{1}-\frac{2 m}{N} \\
B) \quad L=-R+\frac{2 m}{N}, & L \geq 1+m_{1}+J_{1}, \quad J_{2}=0 \\
C) \quad L=m_{1}, & R=\frac{2 m}{N}-m_{1}, \quad J_{1}=J_{2}=0
\end{array}
$$

(or $J_{1} \rightarrow J_{2}, r \rightarrow-r, \frac{2 m}{N} \rightarrow 2 m_{1}-\frac{2 m}{N}$ for series B. In terms of the Dynkin labels these bounds have the following form:

$$
\begin{array}{ll}
\text { A) } & n_{2} \geq n_{1}+1, \quad n_{N+2} \geq n_{N+3}+1 \\
\text { B) } n_{2} \geq n_{1}+1, \quad n_{N+2}=0, \quad n_{N+3}=0 \\
\text { C) } n_{2}=0, & n_{N+2}=0, \quad n_{1}=n_{N+3}=0
\end{array}
$$

$\left(\right.$ or $n_{1} \rightarrow n_{N+3}, n_{2} \rightarrow n_{N+2}$ for series B.)

Notice that for series $\mathrm{C}$ the coefficients above the two odd nodes, and above the first and last nodes must be zero, and for series B one either has the first two coefficients zero, or the last two zero. We will find that for fields on analytic superspaces there is a simple interpretation for these bounds, namely that for fields which carry non-trivial representations of supergroups, all superindices are downstairs.

\section{Reducibility conditions and constraints}

Having indicated the way in which representations of the superconformal group can be carried by superfields on different spaces, we come to the question of when these representations are irreducible. We know that on super Minkowski space the superfields often have to satisfy constraints in order to be irreducible. In this section we review the constraints that UIRs must satisfy on Minkowski and super Minkowski space, and show why no such constraints are required on $(N, p, q)$ analytic superspaces with $p, q \geq 1$. In other words superfields on such analytic superspaces are automatically irreducible. 


\subsection{Verma modules and invariant submodules}

The question of when representations are reducible can be answered with the help of Verma modules (see [36]). A Verma module $V(\lambda)$ with highest weight $\lambda$ is the set spanned by the highest weight vector and all its descendants. A positive root $\alpha$ has a corresponding co-root, $\alpha^{\vee}$ (228), and corresponding Cartan matrix $H_{\alpha}=h_{\alpha \vee}(226)$. It also has an associated raising (lowering) operator, $e_{ \pm \alpha}$ defined by

$$
\left[h, e_{ \pm \alpha}\right]= \pm \alpha(h) e_{ \pm \alpha}
$$

for all elements of the Cartan algebra $h$. A basis for the Verma module $V(\lambda)$ is given by applying all possible lowering operators on the highest weight state

$$
\prod_{\alpha \in \Delta^{+}}\left(e_{-\alpha}\right)^{m_{\alpha}}|\lambda\rangle
$$

where $\Delta^{+}$is the set of positive roots and $m_{\alpha}$ are positive integers. This gives a vector of weight

$$
\lambda-\sum m_{\alpha} \alpha .
$$

This module is reducible if and only if it contains a vector, called a singular vector, which has the characteristic of the highest weight state of another Verma module. In other words we are looking for vectors $|\mu\rangle$ in $V(\lambda)$ which are annihilated by all raising operators $e^{+}$and are thus highest weight state of invariant submodules.

We give some motivations for the formula for finding invariant submodules for $\mathfrak{s l}(n)$. We have $n-1$ positive simple roots, $\alpha_{j}$, with corresponding Cartan matrices $H_{j}$ and corresponding raising and lowering operators $e_{j}^{+}$and $e_{j}^{-}$. These are given by

$$
\begin{aligned}
H_{j} & =\hat{e}_{j j}-\hat{e}_{(j+1)(j+1)} \\
e_{j}^{+} & =\hat{e}_{j(j+1)} \\
e_{j}^{-} & =\hat{e}_{(j+1) j}
\end{aligned}
$$

where $\hat{e}_{j k}$ is the matrix with zeros everywhere except in the $(j k)$ entry where there is a 1 . First consider vectors of the form

$$
|\mu\rangle=\left(e_{j}^{-}\right)^{n}|\lambda\rangle
$$

with weight

$$
\mu=\lambda-n \alpha_{j} .
$$

This is annihilated by all the operators $\left\{e_{i}^{+}: i \neq j\right\}$, since $e_{i}^{+}$commutes with $e_{j}^{-}$for $i \neq j$. One can show that

$$
e_{j}^{+}|\mu\rangle=n\left(n_{j}-n+1\right)|\mu\rangle
$$

where $n_{j}=\left(\lambda, \alpha_{j}^{\vee}\right)$ is the $j$ th Dynkin label for the weight $\lambda$. If we thus choose $n=n_{j}+1$ then $|\mu\rangle$ is annihilated by all raising operators and so $V(\mu)$ is an invariant submodule of $V(\lambda)$.

To find all possible submodules of $V(\lambda)$ we need the concept of a Weyl reflection. For each root $\alpha$ a Weyl reflection $\sigma_{\alpha}$ acts on the weight space as

$$
\sigma_{\alpha}(\lambda)=\lambda-\left(\lambda, \alpha^{\vee}\right) \alpha .
$$


Then the highest weight of the submodule defined above, $\mu=\lambda-\left(n_{j}+1\right) \alpha_{j}$, is just

$$
\mu=\sigma_{\alpha_{j}}(\lambda+\rho)-\rho \quad\left(\lambda+\rho, \alpha^{\vee}\right)>0
$$

where $\rho$ is the weight with Dynkin coefficients $(1, \ldots, 1)$. It is clear that if we repeat this process we will obtain the highest weight of another invariant submodule $\mu^{\prime}=\sigma_{\alpha_{k}} \sigma_{\alpha_{j}}(\lambda+\rho)-\rho$, where $\alpha_{i}, \alpha_{j}$ are simple roots.

In fact the highest weights of all invariant submodules $V(\mu)$ can be obtained by repeated Weyl reflections (see [36]). That is, all highest weights of invariant submodules have the form

$$
\mu=\sigma_{\alpha_{M}} \sigma_{\alpha_{M-1}} \ldots \sigma_{\alpha_{1}}(\lambda+\rho)-\rho
$$

where $\alpha_{p}$ is a sequence of simple positive roots satisfying

$$
m_{p} \equiv\left(\sigma_{\alpha_{p-1}} \sigma_{\alpha_{p-2}} \ldots \sigma_{\alpha_{1}}(\lambda+\rho), \alpha_{p}^{\vee}\right)>0 \quad p=1 \ldots M
$$

The weight vector is given in terms of the $m_{p}$ as

$$
\mu=\lambda-\sum m_{p} \beta_{p}
$$

\subsection{Reducibility conditions on Minkowski space}

Induced representations on non-compact manifolds may also posses invariant subrepresentations. These are essentially the same as above with the caveat that many of the invariant subrepresentations found previously will be automatically zero because of the construction of induced representations. To find the remaining subrepresentations we proceed as follows (the differential operators which one must apply to make irreducible representations of the real conformal group $S U(2,2)$ were found in [37-39]). On Minkowski space we say that a positive root $\beta$ is non-compact if $\mathfrak{g}_{\beta} \subset \mathfrak{n}$ where

$$
\mathfrak{n}=\left\{\left(\begin{array}{ll} 
& \\
\bullet & \bullet \\
\bullet & \bullet
\end{array}\right)\right\}
$$

Then the criteria for finding invariant submodules given in the previous subsection is adapted to the following.

A representation with highest weight $\lambda$ is reducible if for some non-compact root $\beta$ one of the following two conditions holds:

1.) $\quad\left(\lambda+\rho, \beta^{\vee}\right)>0$

2.) $\quad \lambda^{\prime}=\sigma_{\beta}(\lambda+\rho)-\rho$ is dominant integral for $\mathfrak{p}$.

If the first condition is met but not the second, then the representation may still be reducible if $\lambda^{\prime}=\sigma_{\beta}(\lambda+\rho)-\rho$ satisfies these two conditions. If $\lambda^{\prime}$ satisfies the first condition for a non-compact root $\beta^{\prime}$ but not the second condition, then we repeat this process, defining $\lambda^{\prime \prime}=\sigma_{\beta^{\prime}}\left(\lambda^{\prime}+\rho\right)-\rho$ and seeing whether it satisfies any of the conditions. 
Note that the first condition tells us that a state with weight $\lambda^{\prime}=\sigma_{\beta}(\lambda+\rho)-\rho$ can, in principle, be obtained by applying lowering operators $\left(\lambda^{\prime} \prec \lambda\right.$ using the partial ordering defined in the appendix (246)). In the cases we consider this state is annihilated by all raising operators and thus it is the highest weight state of an invariant subrepresentation, $V\left(\lambda^{\prime}\right)$ of $V(\lambda)$ (see previous subsection). When considering representations as fields on a parabolic space some of these subrepresentations are zero automatically, namely those for which $\lambda^{\prime}$ is not dominant with respect to $\mathfrak{p}$. This is because we demand that the fields carry irreducible finite dimensional representations of the parabolic subgroup. Hence we require the second condition. Note also that we only need to consider applying non-compact roots because if we apply compact roots then again the subrepresentation is zero automatically since we are considering irreducible representations of the parabolic subgroup.

We make our representations irreducible by demanding that all invariant subrepresentations are zero. The irreducibility conditions are therefore given by differential operators which take $V(\lambda) \mapsto V_{\lambda^{\prime}}$ (or $V_{\lambda} \mapsto V_{\lambda^{\prime \prime}}$ etc.). Setting these maps to zero will give irreducible representations.

For Minkowski space there are four non-compact roots $\alpha_{2}, \alpha_{2}+\alpha_{1}, \alpha_{2}+\alpha_{3}, \alpha_{1}+\alpha_{2}+\alpha_{3}$ and condition 1 ) says that in order for a representation to be reducible one of the following 4 conditions must hold

$$
\begin{aligned}
n_{2}+1 & >0 \\
n_{1}+n_{2}+2 & >0 \\
n_{2}+n_{3}+2 & >0 \\
n_{1}+n_{2}+n_{3}+3 & >0 .
\end{aligned}
$$

If we put these conditions together with the unitary bounds (see appendix (262) we find that the only reducible representations are those with the following Dynkin labels

$$
\begin{gathered}
(0,0,0) \\
(k,-k-1,0) \\
(0,-k-1, k) \\
\left(n_{1},-n_{1}-n_{3}-2, n_{3}\right)
\end{gathered}
$$

where $k, n_{1}, n_{3}$ are non-negative integers.

Consider the representation with highest weight $\lambda=(k,-k-1,0)$. We find that $\lambda^{\prime}=\sigma_{\alpha_{1}+\alpha_{2}}(\lambda+$ $\rho)-\rho=(k-1,-k-2,1)$ which is dominant integral for $k \geq 0$. Thus the reducibility condition is given by the map

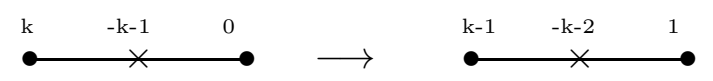

In terms of fields we have $\psi_{\alpha_{1} \ldots \alpha_{k}}$ with the irreducibility condition

$$
\partial_{\dot{\beta}}^{\alpha_{1}} \psi_{\alpha_{1} \ldots \alpha_{k}}=0
$$

and we recognise the usual massless field equations for right handed fields. In a similar way the representation with highest weight $(0,-k-1, k)$ gives left-handed massless fields.

The case $k=0$ is more complicated as $\lambda^{\prime}=(-1,-2,0)$ is not dominant for $\mathfrak{p}$ so condition 2$)$ is not satisfied. We therefore have to apply the same procedure to $\lambda^{\prime}$. We find that $\lambda^{\prime}$ satisfies 
conditions 1) and 2) for the non-compact root $\alpha_{2}+\alpha_{3}$ giving $\lambda^{\prime \prime}=(0,-3,0)$. The reducibility condition is therefore given by

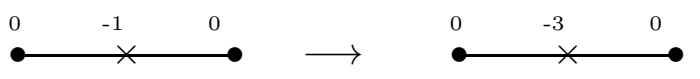

As a field we have a scalar field $\phi$ satisfying the second order equation

$$
\partial_{\alpha \dot{\alpha}} \partial^{\alpha \dot{\alpha}} \phi=0
$$

and we recognise the usual massless scalar equation.

For the representations $\left(n_{1},-n_{1}-n_{3}-2, n_{3}\right)$ we find fields $\psi_{\alpha \ldots \gamma \dot{\alpha} \ldots \dot{\gamma}}$ with $n_{1}$ undotted and $n_{3}$ dotted indices, satisfying the equation

$$
\partial^{\alpha \dot{\alpha}} \psi_{\alpha \beta \ldots \gamma \dot{\alpha} \dot{\beta} \ldots \dot{\gamma}}=0
$$

if $n_{1}, n_{3}>0$. In the case $n_{1}=0$ or $n_{3}=0$ we find $\lambda^{\prime}$ does not satisfy condition 1$)$ and thus the representations are irreducible (without any conditions).

\subsection{The supersymmetric case}

We briefly adapt some of the discussion in section 4.1 to the supergroup $\mathfrak{s l}(4 \mid N)$ following [4]. Again, for each positive root $\alpha$ we have a corresponding raising (lowering) operator $e_{ \pm \alpha}$ defined by

$$
\left[h, e_{ \pm \alpha}\right]= \pm \alpha(h) e_{ \pm \alpha}
$$

for all elements of the Cartan algebra $h$. The Verma module $V(\lambda)$ is obtained by applying all possible combinations of lowering operators to the highest weight state and this module is reducible if and only if it contains an invariant submodule $V(\mu)$ with highest weight $\mu$. These are known as singular vectors. So we are looking for vectors $|\mu\rangle$ in $V(\lambda)$ which are annihilated by all raising operators $e^{+}$.

Again we will motivate the discussion by considering simple roots. For even simple roots we obtain, as previously, that the weight $\mu=\lambda-\left(n_{j}+1\right) \alpha_{j}$ is the highest weight of an invariant submodule. For odd simple roots $\beta$, consider the state $|\mu\rangle=e_{\beta}^{-}|\lambda\rangle$ (note we can only apply the lowering operator once as it is odd.) Then

$$
e_{\beta}^{+}|\mu\rangle=H_{\beta}|\lambda\rangle .
$$

So this is zero if and only if $H_{\beta}|\lambda\rangle=0$.

We define Weyl reflections for superalgebras. For even roots they are defined as in (80) whereas for odd roots $\beta$ we define

$$
\sigma_{\beta}(\lambda)=\lambda-\beta, \quad(\beta, \beta)=0 .
$$

We also define the weight $\rho$ to have Dynkin coefficients $(1,0,1,1, \ldots, 1,0,1)$ so it has 1 's above all even nodes and 0's above the odd nodes in the Dynkin diagram. We see that the invariant submodules have highest weights $\mu$ where

$$
\mu=\sigma_{\alpha}(\lambda+\rho)-\rho \quad\left(\lambda+\rho, \alpha^{\vee}\right)>0 \quad(\alpha \text { even }) \quad\left(\lambda+\rho, \alpha^{\vee}\right)=0 \quad(\alpha \text { odd }) .
$$

Once again, all invariant submodules are obtained by repeated Weyl reflections. 


\subsection{Reducibility constraints for fields on super Minkowski space}

In this section we review the constraints fields on super Minkowski space or harmonic superspace must satisfy in order to carry irreducible representations of the superconformal group.

We define non-compact positive roots $\beta$ (even or odd) to be those such that $\mathfrak{g}_{\beta} \subset \mathfrak{n}$ where $\mathfrak{n}$ is the set of matrices of the form

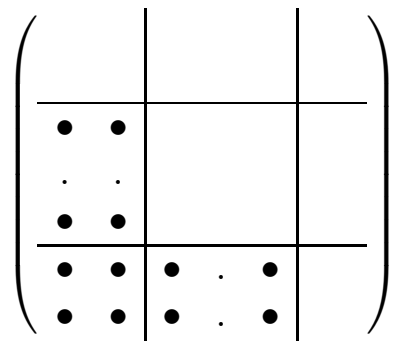

The reducibility conditions in the bosonic case (86) are straightforwardly modified for the supersymmetric case [4]. Field representations on super Minkowski space with highest weight $\lambda$ are reducible if for some $\beta$, the following two conditions are met:

$$
\text { 1.) } \quad\left(\lambda+\rho, \beta^{\vee}\right)=0(\beta \text { odd }) \quad\left(\lambda+\rho, \beta^{\vee}\right)>0(\beta \text { even })
$$

2.) $\sigma_{\beta}(\lambda+\rho)-\rho$ is dominant integral for $\mathfrak{p}$.

The weight $\lambda^{\prime}=\sigma_{\beta}(\lambda+\rho)-\rho$ is the highest weight of an invariant subrepresentation. If condition 1 ) is met but condition 2) is not then one checks to see if $\lambda^{\prime}=\sigma_{\beta}(\lambda+\rho)-\rho$ satisfies similar conditions. Repeat this process if $\lambda^{\prime}$ only satisfies condition 1$)$. The conditions one must impose to make the representations irreducible are given by differential operators which map $V_{\lambda} \rightarrow V_{\lambda^{\prime}}$ (or $V_{\lambda} \rightarrow V_{\lambda^{\prime \prime}}$ etc.) where $V_{\lambda}$ denote the representation with highest weight $\lambda$. The differential operators correspond to the infinitesimal Lie algebra element $\mathfrak{g}_{-\beta}$ since $\mathfrak{g}_{-\beta}$ takes a state with weight $\lambda$ to one with weight $\lambda^{\prime}$.

So for super Minkowski space we have $4 N$ non-compact odd roots and 4 non-compact even roots so there are $4 N+4$ possible reducibility conditions. Condition 1) becomes (for the $4 N$ odd roots)

$$
\begin{aligned}
n_{2}+\sum_{i=1}^{a} n_{2+i}+a & =0 \\
n_{2}+\sum_{i=1}^{a} n_{2+i}+a-n_{1}-1 & =0 \\
n_{2+N}+\sum_{i=1}^{a} n_{2+i}+a & =0 \\
n_{2+N}+\sum_{i=1}^{a} n_{2+i}+a-n_{3+N}-1 & =0
\end{aligned}
$$

where $a=0 \ldots N-1$. Putting these together with the unitary bounds (70) we find that the 
equations can only be satisfied for $a=0$ giving

\begin{tabular}{cccc}
$\operatorname{root} \beta$ & condition & $\lambda$ & $\lambda^{\prime}$ \\
\hline$\alpha_{2}$ & $n_{1}=n_{2}=0$ & $\left(0,0, n_{3}, \ldots, n_{N+3}\right)$ & $\left(1,0, n_{3}+1, n_{4}, \ldots, n_{N+3}\right)$ \\
$\alpha_{1}+\alpha_{2}$ & $n_{2}=n_{1}+1$ & $\left(n_{1}, n_{1}+1, n_{3}, \ldots, n_{N+3}\right)$ & $\left(n_{1}-1, n_{1}, n_{3}+1, n_{4}, \ldots, n_{N+3}\right)$ \\
$\alpha_{N+2}$ & $n_{2+N}=n_{3+N}=0$ & $\left(n_{1}, \ldots, n_{N+1}, 0,0\right)$ & $\left(n_{1}, \ldots, n_{N}, n_{N+1}+1,0,1\right)$ \\
$\alpha_{N+2}+\alpha_{N+3}$ & $n_{2+N}=n_{3+N}+1$ & $\left(n_{1}, \ldots, n_{N+1}, n_{N+3}+1, n_{N+3}\right)$ & $\left(n_{1}, \ldots, n_{N+1}+1, n_{N+3}, n_{N+3}-1\right)$.
\end{tabular}

Furthermore for $N>0$ the conditions coming from the even compact roots are incompatible with the unitary bounds and therefore these conditions above are the only possible shortening conditions. Corresponding to each of these conditions there are the following irreducibility conditions

$$
\begin{array}{rll}
\alpha_{2} & : & \left(D_{\alpha i} W_{j k \ldots}\right)_{\left(n_{3}+1, n_{4}, \ldots\right)}=0 \\
\alpha_{1}+\alpha_{2} & : \quad & \left(D_{i}^{\alpha} W_{j k \ldots l} \ldots \ldots \gamma\right)_{\left(n_{3}+1, n_{4}, \ldots\right)}=0
\end{array}
$$

where the subscript numbers tell us which $S U(N)$ representation to take. There are similar conjugate conditions for the other two cases. If $n_{1}=0$ the second equation does not apply. This is because condition 2) above is not met i.e. $\lambda^{\prime}$ is not dominant for $\mathfrak{p}$ and thus we have to consider reducibility conditions for $\lambda^{\prime}$. One finds that the conditions 1) and 2) are met for $\beta=\alpha_{1}$ giving

$$
\begin{array}{ccc}
\lambda^{\prime} & \longrightarrow & \lambda^{\prime \prime} \\
\left(-1,0, n_{3}+1, n_{4}, \ldots, n_{N+3}\right) & \longrightarrow & \left(0,0, n_{3}+2, n_{4}, \ldots, n_{N+3}\right) .
\end{array}
$$

This leads to the second order reducibility condition $\left(V_{\lambda} \rightarrow V_{\lambda^{\prime \prime}}\right)$ for fields on Minkowski space

$$
D_{\alpha(i} D_{j}^{\alpha} W_{k) l \ldots m}=0 .
$$

One gets a similar (complex conjugate) equation if $n_{3+N}=0$ in the fourth reducibility equation. Note that all these results are consistent with the equations satisfied by the massless multiplets in section 2.1, and they agree with the results found in [33] for fields on harmonic superspace.

\subsection{Fields on analytic superspace}

The irreducibility conditions lift up straightforwardly to $(N, p, q)$ harmonic superspace under the fibration (52), since the fibres are compact (the Bott-Borel-Weil theorem can be applied on the fibres.) By this we mean that the reducibility condition remains $V_{\lambda} \rightarrow V_{\lambda^{\prime}}$. The differential operators which implement this on harmonic superspace can be found in [33] using a generalised harmonic superspace with internal space $B \backslash S L(N)$.

If we now consider pushing these fields down to $(N, p, q)$ analytic superspace under the fibration (55) (with $p, q \geq 1$ ) we find that the reducibility conditions are satisfied automatically. To see this we consider the respective isotropy groups of super Minkowski space, and analytic 
superspace $(42,53)$
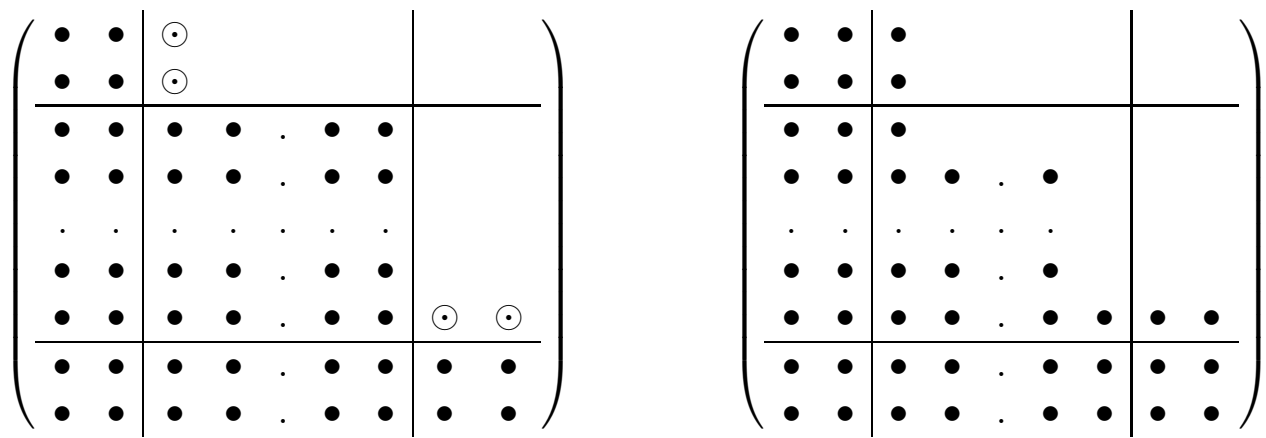

In this diagram we use $(N, 1,1)$ analytic superspace for illustration, but the result is true for any $(N, p, q)$ space with $p, q \geq 1$. The circles correspond to the subspaces $\mathfrak{g}_{-\beta}$ for $\beta \in\left\{\alpha_{2}, \alpha_{1}+\right.$ $\left.\alpha_{2}, \alpha_{N+2}, \alpha_{N+2}+\alpha_{N+3}\right\}$, the roots associated with the reducibility conditions (109). On super Minkowski space the infinitesimal Lie algebra elements $\mathfrak{g}_{-\beta}$ lie in $\mathfrak{n}^{-}$, the generator of translations in super Minkowski space. And so the constraint $V_{\lambda} \rightarrow V_{\lambda^{\prime}}$ is a differential operator. On any $(N, p, q)$ analytic superspace with $p, q \geq 1$ however, the subspaces $\mathfrak{g}_{-\beta}$ lie in the isotropy group which means the map $V_{\lambda} \rightarrow V_{\lambda^{\prime}}$ is no longer a differential operator but a rotation of the isotropy group. But since we are only considering finite irreducible representations of the isotropy group, the constraint $V_{\lambda^{\prime}}=0$ will be solved automatically as otherwise $V_{\lambda^{\prime}}$ would restrict to a subrepresentation of the isotropy group.

We conclude that unitary irreducible representations of the superconformal group are given by unconstrained fields on analytic superspace.

\section{$5 \quad$ Fields with superindices}

We have argued that any representation of the superconformal group can be given as a holomorphic field on any superflag space (except supertwistor spaces). We will here give some explicit examples of fields which transform under supergroups in analytic superspace. We will see that they do indeed give irreducible representations.

\subsection{Superindices}

The fields on $(N, p, q)$ analytic superspace will carry irreducible representations of the supergroups $\mathfrak{s l}(2 \mid p)$ and $\mathfrak{s l}(2 \mid q)$. All finite dimensional irreducible representations of $\mathfrak{s l}(N)$ can be described as symmetrised tensor products of the fundamental representation. Young Tableaux provide a useful way of describing these symmetries. It is true for $\mathfrak{s l}(r \mid s)$ that all finite-dimensional representations with integer Dynkin labels can be obtained as tensor products of the fundamental (and anti-fundamental) representation [7]. We will deal with other finite dimensional representations later. Let $g_{B}^{A}$ be a matrix in $\mathfrak{s l}(r \mid s)$ so that $A=(\alpha \mid a)$, where $\alpha$ runs from 1 to $r$ and $a$ runs from $r+1$ to $r+s$, is a superindex. We define the following generalised 
symmetrisation rules for the superindices

$$
(A B)=\left\{\begin{array}{ll}
(\alpha \beta) & A=\alpha, B=\beta \\
(\alpha b) & A=\alpha, B=b \\
(a \beta) & A=a, B=\beta \\
{[a b]} & A=a, B=b
\end{array} \quad[A B]= \begin{cases}{[\alpha \beta]} & A=\alpha, B=\beta \\
{[\alpha b]} & A=\alpha, B=b \\
{[a \beta]} & A=a, B=\beta \\
(a b) & A=a, B=b\end{cases}\right.
$$

Indices in the range $A=1,2$ are called 'even' and those in the range $A=3,4, \ldots, 2+s$ are called 'odd' since they swap with a minus sign. So irreducible representations of $\mathfrak{s l}(r \mid s)$ are carried by tensors $W^{A B \ldots C}$ with $m \mathfrak{s l}(r \mid s)$ indices which satisfy various symmetry properties indicated by a super Young Tableau with $m$ boxes [40]. The super Young Tableau gives the symmetries in the usual way (rows corresponding to symmetrised indices and columns corresponding to antisymmetrised indices) except that the definition of (anti)symmetry is modified to generalised (anti)symmetry described above. We can convert this Young Tableau into a Dynkin diagram by finding the highest weight state as in the bosonic case. We will do this explicitly for the group $\mathfrak{s l}(2 \mid s)$ which we will use later.

The highest weight state $W^{1 \ldots 1} 2 \ldots 2 \ldots$ is found by putting numbers in the boxes of the Young tableau as follows

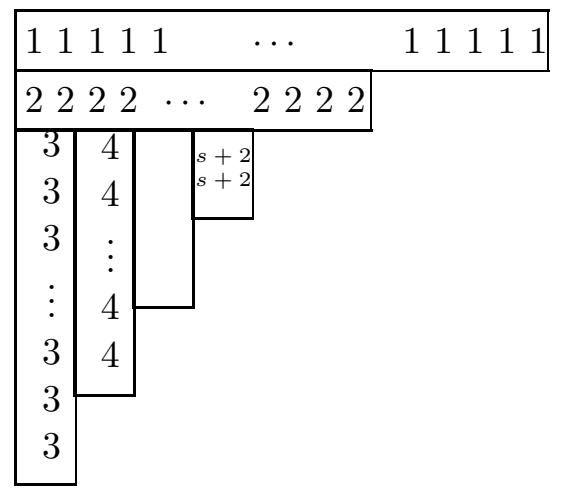

Unlike in the purely bosonic case, however, contravariant and covariant representations are inequivalent as there is no invariant $\varepsilon$ tensor. Contravariant tensors (which correspond to downstairs indices) have highest weight states given by

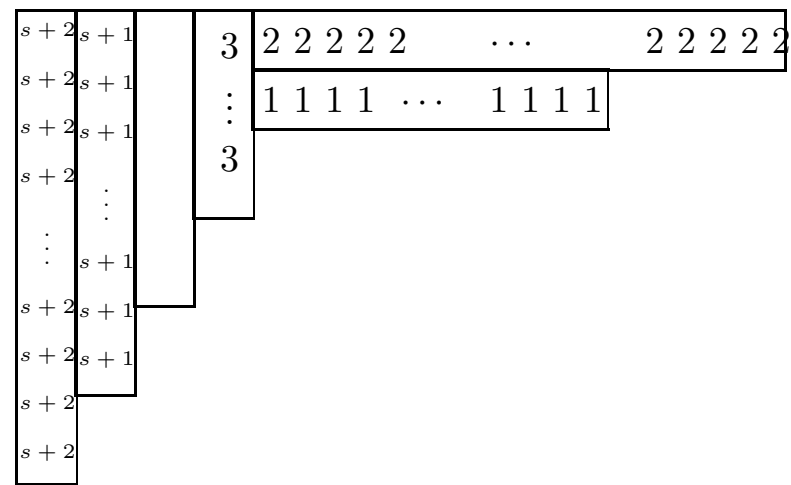

where there are $m_{1} 1$ 's, $m_{2} 2$ 's etc. Then from the definition of the Dynkin coefficients $n_{i}$, and 
from (58) we obtain

$$
\begin{aligned}
m_{s+2}-m_{s+1} & =n_{s+1} \\
m_{s+1}-m_{s} & =n_{s} \\
& \vdots \\
m_{4}-m_{3} & =n_{3} \\
m_{3}+m_{2} & =n_{2} \\
m_{2}-m_{1} & =n_{1} .
\end{aligned}
$$

Since the highest weight state determines the irreducible representation of $\mathfrak{s l}(2 \mid s)$ uniquely, we can have different diagrams which correspond to the same representation. In particular, given a diagram with $m_{1} \neq 0$ we can instead take the diagram with $m_{i}$ replaced by $m_{i}^{\prime}$, where

$$
\begin{array}{rlc}
m_{1}^{\prime} & = & 0 \\
m_{2}^{\prime} & = & m_{2}-m_{1} \\
m_{3}^{\prime} & = & m_{3}+m_{1} \\
& \vdots & \\
m_{s+2}^{\prime} & = & m_{s+2}+m_{1}
\end{array}
$$

and this will have the same Dynkin coefficients and thus correspond to the same representation. Thus for representations of $\mathfrak{s l}(2 \mid s)$ we can assume without any loss of generality that $m_{1}=0$. However, this is not the case when we are considering representations of $\mathfrak{g l}(2 \mid s)$.

Note that if we are given the Dynkin weights $n_{i} \geq 0$ we will have a contravariant tensor representation provided $m_{3} \geq 1$ or $m_{2}=m_{3}=0$ (these are simply conditions for the Young Tableau to have the correct shape). In terms of Dynkin coefficients this is the condition

$$
n_{2} \geq n_{1}+1 \text { or } n_{1}=n_{2}=0
$$

On comparing this condition with the unitarity conditions (70) one can see that fields on analytic superspace will carry contravariant representations.

There is some choice in the way the contravariant tensors transform. A field $W^{A}$ in the fundamental representation transforms as $W^{A} \mapsto g^{A}{ }_{B} W^{B}$. Then a contravariant tensor $V_{A}$ can be chosen to transform so that either $V_{A} W^{A}$ is invariant or $V^{A} W_{A}$ is invariant (in general they won't both be invariant). In the first case $V_{A} \mapsto V_{B}\left(g^{-1}\right)^{B} A$ as one might expect. In the second case however

$$
V_{A} \mapsto V_{B}\left(g^{-1}\right)^{B}{ }_{A}(-1)^{(A+B)}
$$

where $(-1)^{(A+B)}$ is 1 if both $\mathrm{A}$ and $\mathrm{B}$ are even or if both are odd, and is -1 otherwise.

\subsection{Quasi-tensors}

Recall that finite dimensional representations of simple supergroups may have non-integral numbers above the odd nodes of the Dynkin diagram. It is not possible to accommodate such representations as ordinary tensors since these must clearly have integer Dynkin coefficients from (118). One must introduce the concept of 'quasi-tensors' [14] to accommodate these representations. 
Consider representations of $\mathfrak{s l}(2 \mid s)$ with Dynkin coefficients $\left[n_{1}, \ldots, n_{s+1}\right]$. As mentioned, from unitarity we are only interested in representations with downstairs superindices, whose Dynkin labels satisfy $n_{2} \geq n_{1}+1$ or $n_{1}=n_{2}=0$ (120). In fact, if we fix $n_{1}, n_{3}, n_{4}, \ldots, n_{s+1}$ and vary $n_{2}$ we find that all representations for which $n_{2}>n_{1}+1$ have the same dimension. These are called typical representations in the literature [34]. The representations with $n_{2}=n_{1}+1$ and $n_{1}=n_{2}=0$ are known as 'atypical' representations and have a smaller dimension (the latter case being the trivial one dimensional case). The easiest way to see this is to consider the representations acting on holomorphic sections of the parabolic space obtained by putting a cross through the single odd node of the Dynkin diagram for $\mathfrak{s l}(2 \mid s)$ :

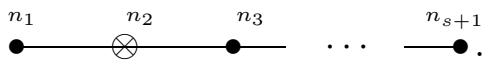

This space has purely odd coordinates. The typical representations are given by unconstrained tensor superfields on this space (and hence are all of the same dimension for given Dynkin labels $\left.n_{1}, n_{3}, \ldots, n_{s+1}\right)$, whereas the atypical representations must satisfy constraints in order to be irreducible. These are similar to the constraints found for representations on super Minkowski space in section 4.4 and indeed the proof goes through using Verma modules in a similar way.

Now given that these atypical representations all have the same dimension, we can represent them abstractly by the simplest possible such tensor, together with a real number to give the value of the odd Dynkin label. Specifically the $\mathfrak{s l}(2 \mid s)$ representation with Dynkin labels $\left[n_{1}, n_{1}+\right.$ $\left.2+p, n_{3}, \ldots, n_{s+1}\right]$ we represent by the ordinary tensor specified by labels $\left[n_{1}, n_{1}+2, n_{3}, \ldots, n_{s+1}\right]$ (we can find the corresponding tensor by following the rules in section 5.1) together with the number $p$. Since we know how this transforms for all integer values of $p \geq 0$, we can then continue $p$ to real (or even complex) values to find out how the quasi-tensors transform.

The simplest example which illustrates this is given in $\mathfrak{s l}(2 \mid 1)$ [14]. Consider representations with Dynkin labels $[0, p+2]$. For all integer values of $p \geq-2$ this representation can be given by a tensor $\mathcal{O}_{A B C_{1} \ldots C_{p}}$ which is (generalised) antisymmetric on all $n=p+2$ indices. It is not difficult to check that all such tensors do indeed have the same number of components for $p=0,1,2, \ldots$. We now define the quasi-tensor $\mathcal{O}_{A B}[p]$ to be the object with components

$$
\begin{aligned}
\mathcal{O}_{33}[p] & =\mathcal{O}_{3333 \ldots} \\
\mathcal{O}_{\alpha 3}[p] & =\mathcal{O}_{\alpha 333 \ldots} \\
\mathcal{O}_{\alpha \beta}[p] & =\mathcal{O}_{\alpha \beta 33 \ldots}
\end{aligned}
$$

All of the other components of $\mathcal{O}$ vanish by antisymmetry of the even indices. If we take $\mathfrak{s l}(2 \mid 1)$ to act from the left, we find its action on these tensors to be

$$
\begin{aligned}
\delta \mathcal{O}_{33}[p] & =(p+2) A_{3}{ }^{\alpha} \mathcal{O}_{\alpha 3}[p]+(p+2) A_{3}{ }^{3} \mathcal{O}[p] \\
\delta \mathcal{O}_{\alpha 3}[p] & =\hat{A}_{\alpha}{ }^{\beta} \mathcal{O}_{\beta 3}[p]+\left(p+\frac{3}{2}\right) A_{3}{ }^{3} \mathcal{O}_{\alpha 3}[p]+A_{\alpha}{ }^{3} \mathcal{O}_{33}[p]+(p+1) A_{3}{ }^{\beta} \mathcal{O}_{\alpha \beta}[p] \\
\delta \mathcal{O}_{\alpha \beta}[p] & =(p+1) A_{3}{ }^{3} \mathcal{O}_{\alpha \beta}[p]-2 A_{[\alpha}{ }^{3} \mathcal{O}_{\beta] 3}[p]
\end{aligned}
$$

where $A_{A}{ }^{B} \in \mathfrak{s l}(2 \mid 1)$ and $\hat{A}_{\alpha}{ }^{\beta}$ is traceless. Now this formula makes sense for arbitrary values of $p$ (even complex) and it is straightforward to check that one still has a representation of the 
algebra. In the context of unitary representations of the superconformal group we are interested in $p$ real and $p \geq-1$ or $p=-2$. For $p=-1$ the representation becomes reducible, although not completely reducible. The components $\left(\mathcal{O}_{33}[-1], \mathcal{O}_{\alpha 3}[-1]\right)$ transform under a subrepresentation, while the quotient representation in this case is just a singlet. For the case $p=-2$ we see that the component $O_{33}[-2]$ is invariant and we obtain the trivial representation. These two cases are atypical representations whereas for $p>-1$ the representations are typical.

\subsection{Representations on various superspaces}

We are now in a position to give any representation as a superfield on different superspaces. Note that unitary irreducible representations can not be carried by holomorphic fields on super twistor space. On super twistor space (56) we would obtain fields transforming under the representation of the semisimple part of the Levi subalgebra $\mathfrak{l}_{S}=\mathfrak{s l}(3 \mid N)$ with the following Dynkin diagram

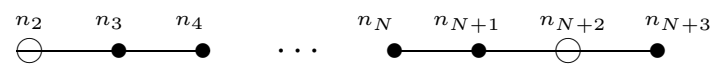

This diagram is not in the distinguished basis as it contains two odd nodes. If we rearrange this in terms of the distinguished set of simple roots of $\mathfrak{s l}(3 \mid N)$ we get

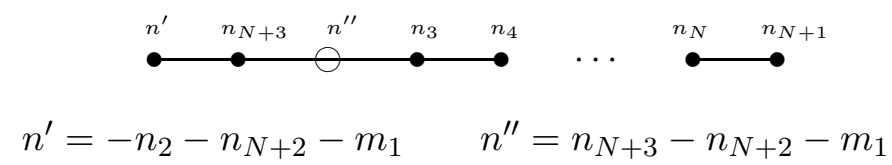

where $m_{1}$ is defined in (62). We see that the coefficient $n^{\prime}$ is negative and therefore the representation is not finite dimensional (recall from the paragraph following (57) that a representation is finite dimensional if and only if all the distinguished even coefficients are positive). We therefore exclude this space from our analysis. We also exclude dual projective twistor space that has one cross on the right hand node of the Dynkin diagram for $S L(4 \mid N)$, and ambitwistor space, which has crosses on both extremal nodes, for the same reason. Note that the problem with these spaces is the same as in the bosonic case using twistor space (section A.6). One must presumably use higher cohomology to treat these cases as with twistor space.

We can, however give any unitary representation of the superconformal group as a holomorphic superfield on any other superflag space. These superfields will sometimes have to satisfy constraints to make them irreducible representations. However, on analytic superspaces the fields require no constraints as shown in section 4 .

For the reasons just given, we will mainly be interested in analytic superspaces. A unitary representation on $(N, p, q)$ analytic superspace has Dynkin diagram

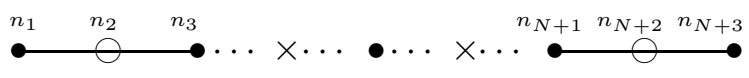

The fields transform under the supergroups $\mathfrak{s l}(2 \mid p)$ and $\mathfrak{s l}(2 \mid q)$ under the representation given by the first $p+1$ nodes and the last $q+1$ nodes. But as mentioned, the unitarity conditions (70) tell us that these are either trivial representations or contravariant representations 
(compare with (120).) In other words, on analytic superspaces the unitary representations are characterised by the fields with downstairs supergroup indices. In fact this can be made into a general statement about any superspace (except the twistor spaces mentioned above): the unitary representations are precisely those fields with downstairs superindices.

\section{$5.4 N=2$ analytic superspace}

We now look at some specific examples on complex $N=2$ analytic superspace. This has the following Dynkin diagram

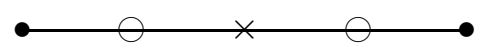

with corresponding parabolic subgroup consisting of matrices of the form

$$
\left(\begin{array}{ll}
a_{B}^{A} & 0 \\
c_{A^{\prime} B} & d_{A^{\prime}}^{B^{\prime}}
\end{array}\right)
$$

where each entry is a $(2 \mid 1) \times(2 \mid 1)$ matrix. The Levi subalgebra (under which our fields transform) is $\mathfrak{s l}(2 \mid 1) \oplus \mathfrak{s l}(2 \mid 1) \oplus \mathbb{C}$ (corresponding to the block diagonals), where the first $\mathfrak{s l}(2 \mid 1)$ subalgebra is carried by un-primed indices, and the second by primed indices.

One needs two coordinate charts $U$ and $U^{\prime}$, with coordinates $X$ and $X^{\prime}$, to cover the whole of complex analytic superspace. Coset representatives for each of these charts can be given as follows

$$
\begin{aligned}
& s_{1}(X)=\left(\begin{array}{c|cc|c}
1_{2} & 0 & \lambda & x \\
\hline 0 & 1 & y & \pi \\
0 & 0 & 1 & 0 \\
\hline 0 & 0 & 0 & 1
\end{array}\right) \\
& s_{2}\left(X^{\prime}\right)=\left(\begin{array}{c|cc|c}
1_{2} & \lambda^{\prime} & 0 & x^{\prime} \\
\hline 0 & y^{\prime} & 1 & \pi^{\prime} \\
0 & 1 & 0 & 0 \\
\hline 0 & 0 & 0 & 1_{2}
\end{array}\right) .
\end{aligned}
$$

These are related by a superconformal transformation, i.e.

$$
s_{2}\left(X^{\prime}\right)=s_{1}\left(X^{\prime}\right) K, \quad K=\left(\begin{array}{c|cc|c}
1_{2} & 0 & 0 & 0 \\
\hline 0 & 0 & 1 & 0 \\
0 & 1 & 0 & 0 \\
\hline 0 & 0 & 0 & 1_{2}
\end{array}\right)
$$

By performing a compensating isotropy group transformation, $\left.s_{2}\left(X^{\prime}\right)=h s_{1}\left(X\left(X^{\prime}\right)\right)\right)$ we can relate the two sets of coordinates on $U \cap U^{\prime}$ :

$$
\left(\begin{array}{c|cc|c}
1_{2} & \lambda^{\prime} & 0 & x^{\prime} \\
\hline 0 & y^{\prime} & 1 & \pi^{\prime} \\
0 & 1 & 0 & 0 \\
\hline 0 & 0 & 0 & 1_{2}
\end{array}\right)=\left(\begin{array}{c|cc|c}
1_{2} & \lambda^{\prime} & 0 & 0 \\
\hline 0 & y^{\prime} & 0 & 0 \\
0 & 1 & -\frac{1}{y^{\prime}} & -\frac{\pi^{\prime}}{y^{\prime}} \\
\hline 0 & 0 & 0 & 1_{2}
\end{array}\right)\left(\begin{array}{c|cc|c}
1_{2} & 0 & -\frac{\lambda^{\prime}}{y^{\prime}} & x^{\prime}-\frac{\lambda^{\prime} \pi^{\prime}}{y^{\prime}} \\
\hline 0 & 1 & \frac{1}{y^{\prime}} & \frac{\pi^{\prime}}{y^{\prime}} \\
0 & 0 & 1 & 0 \\
\hline 0 & 0 & 0 & 1_{2}
\end{array}\right)
$$


giving

$$
\begin{aligned}
x & =x^{\prime}-\frac{\lambda^{\prime} \pi^{\prime}}{y^{\prime}} \\
\lambda & =-\frac{\lambda^{\prime}}{y^{\prime}} \\
\pi & =\frac{\pi^{\prime}}{y^{\prime}} \\
y & =\frac{1}{y^{\prime}}
\end{aligned}
$$

Requiring our fields to be holomorphic on both patches puts restrictions on the fields, which are equivalent to the constraints on Minkowski space.

We use the formalism of induced representations (254-257), representations of parabolic subgroups, and superindices outlined above to find the transformation of fields. Define $f(X)=$ $F\left(s_{1}(X)\right)$ and $f^{\prime}\left(X^{\prime}\right)=F\left(s_{2}\left(X^{\prime}\right)\right)$, then under the superconformal transformation mapping $X \mapsto X^{\prime}$ we have

$$
f(X) \mapsto f^{\prime}\left(X^{\prime}\right)=R(h) f(X)
$$

where $h$ is given in (137). However only the Levi part of $h$

$$
l=\left(\begin{array}{c|cc|c}
1_{2} & \lambda^{\prime} & 0 & 0 \\
\hline 0 & y^{\prime} & 0 & 0 \\
0 & 0 & -\frac{1}{y^{\prime}} & -\frac{\pi^{\prime}}{y^{\prime}} \\
\hline 0 & 0 & 0 & 1_{2}
\end{array}\right)=\left(\begin{array}{c|cc|c}
1_{2} & -\frac{\lambda}{y} & 0 & 0 \\
\hline 0 & \frac{1}{y} & 0 & 0 \\
0 & 0 & -y & -\pi \\
\hline 0 & 0 & 0 & 1_{2}
\end{array}\right)
$$

acts non-trivially. Comparing with (133) we find

$$
a_{B}^{A}=\left(\begin{array}{c|c}
1_{2} & -\frac{\lambda}{y} \\
\hline 0 & \frac{1}{y}
\end{array}\right) \quad d_{A^{\prime}} B^{\prime}=\left(\begin{array}{c|c}
-y & -\pi \\
\hline 0 & 1_{2}
\end{array}\right) .
$$

For a representation with Dynkin coefficients $n_{i}$ we have the Dynkin diagram

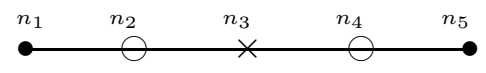

find that

$$
R(h)=y^{-Q} R^{\left(n_{1}, n_{2}\right)}\left(a_{B}^{A}\right) R^{\left(n_{4}, n_{5}\right)}\left(d_{A^{\prime}}^{B^{\prime}}\right)
$$

where $R^{\left(n_{1}, n_{2}\right)}$ and $R^{\left(n_{4}, n_{5}\right)}$ are the representations of $S L(2 \mid 1)$ with Dynkin diagrams

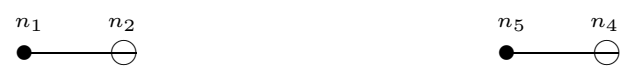

respectively, extended to a representation of $G L(2 \mid 2)$ in the natural way. By considering the corresponding Cartan generators acting on the highest weight state one can show that

$$
Q=-n_{1}+n_{2}+n_{3}+n_{4}-n_{5}=L-J_{1}-J_{2}
$$

in the case that the supergroup representations are contravariant, and is generated by the Cartan matrix $\operatorname{diag}(-1,0|0,0| 0,1)$.

To find the representations $R^{\left(n_{1}, n_{2}\right)}$ and $R^{\left(n_{4}, n_{5}\right)}$ one first converts the Dynkin diagrams into Young Tableaux. From the unitary bounds (70) and the condition (120) we know that we will always obtain trivial or contravariant representations of both the $S L(2 \mid 1)$ subgroups. There is some choice as to how these transform (see discussion in the previous subsection.) We will choose 
our fields so as to transform consistently with $d X^{A A^{\prime}}$ and $\partial / \partial X^{A A^{\prime}}$. Under the transformation (138) we obtain

$$
\begin{aligned}
& \left(\begin{array}{ll}
d \lambda^{\prime} & d y^{\prime} \\
d s^{\prime} & d \pi^{\prime}
\end{array}\right)=\left(\begin{array}{cc}
1 & \frac{-\lambda}{y} \\
0 & \frac{1}{y}
\end{array}\right)\left(\begin{array}{ll}
d \lambda & d y \\
d s & d \pi
\end{array}\right)\left(\begin{array}{cc}
-\frac{1}{y} & -\frac{\pi}{y} \\
0 & 1
\end{array}\right) \\
& \left(\begin{array}{ll}
\partial_{\lambda^{\prime}} & \partial_{y^{\prime}} \\
\partial_{s^{\prime}} & \partial_{\pi^{\prime}}
\end{array}\right)=\left(\begin{array}{cc}
-y & -\pi \\
0 & 1
\end{array}\right)\left(\begin{array}{ll}
\partial_{\lambda} & \partial_{y} \\
\partial_{s} & \partial_{\pi}
\end{array}\right)\left(\begin{array}{cc}
1 & -\lambda \\
0 & y
\end{array}\right)
\end{aligned}
$$

which we can rewrite

$$
\begin{aligned}
d X^{\prime B B^{\prime}} & =a^{B}{ }_{A} d X^{A A^{\prime}}\left(d^{-1}\right)_{A^{\prime}} B^{\prime} \\
\partial_{A^{\prime} A}^{\prime} & =d_{A^{\prime}} B^{\prime} \partial_{B^{\prime} B} \tilde{a}_{A}^{B} .
\end{aligned}
$$

where we define

$$
\tilde{a}_{A}^{B}=\left(a^{-1}\right)_{A}^{B}(-1)^{(A+B)} .
$$

We thus define tensors to transform as follows

$$
\begin{aligned}
W^{A} & \mapsto a_{B}^{A} W^{B} & W^{A^{\prime}} & \mapsto W^{B^{\prime}}\left(d^{-1}\right)_{B^{\prime}} A^{\prime} \\
W_{A} & \mapsto W_{B} \tilde{a}^{B}{ }_{A} & W_{A^{\prime}} & \mapsto d_{A^{\prime}} B^{\prime} W_{B^{\prime}}
\end{aligned}
$$

and we see that the combinations $W^{A} V_{A}$ and $W^{A^{\prime}} V_{A^{\prime}}$ are scalars, whereas $V_{A} W^{A}$ and $V_{A^{\prime}} W^{A^{\prime}}$ are not.

In section 2.3 we gave a few examples of $N=2$ supermultiplets as fields on analytic superspace. We look at these examples now in more detail. The simplest example of a field on analytic superspace is that of the hypermultiplet. The Dynkin diagram for this representation on analytic superspace

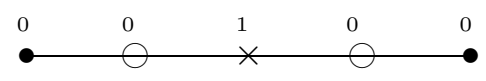

tells us that it has no superindices (since $n_{1}=n_{2}=n_{4}=n_{5}=0$ ) and that $Q=1$ (145). Thus from $(139,143)$ it is a field $W$ which transforms as

$$
W(X) \mapsto W^{\prime}\left(X^{\prime}\right)=\frac{1}{y} W(X)
$$

under the transformation $X \mapsto X^{\prime}(138)$. Demanding that $W(X)$ and $W^{\prime}\left(X^{\prime}\right)$ are both holomorphic in their variables leaves us with the component fields of the hypermultiplet

$$
\begin{aligned}
W(x, \lambda, \pi, y) & =\varphi_{1}(x)+y \varphi_{2}(x)+\lambda^{\alpha} \psi_{\alpha}(x) \\
& +\pi^{\dot{\alpha}} \chi_{\dot{\alpha}}(x)-\lambda^{\alpha} \pi^{\dot{\alpha}} \partial_{\alpha \dot{\alpha}} \varphi_{2} \\
W^{\prime}\left(x^{\prime}, \lambda^{\prime}, \pi^{\prime}, y^{\prime}\right) & =\varphi_{2}\left(x^{\prime}\right)+y^{\prime} \varphi_{1}\left(x^{\prime}\right)-\lambda^{\prime \alpha} \psi_{\alpha}\left(x^{\prime}\right) \\
& +\pi^{\prime \dot{\alpha}} \chi_{\dot{\alpha}}\left(x^{\prime}\right)-\lambda^{\prime \alpha} \pi^{\prime \dot{\alpha}} \partial_{\alpha \dot{\alpha}} \varphi_{1}
\end{aligned}
$$

and with the component fields $\left(\varphi_{1}, \varphi_{2}, \psi, \chi\right)$ all satisfying their equations of motion [19].

Next consider the $N=2$ on-shell Maxwell multiplet, which is usually given as a chiral field in super Minkowski space. It has dilation weight 1 , R-charge -1 and all other quantum numbers are 0 and thus has the following Dynkin diagram as a field on analytic superspace

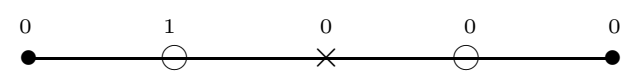


We can read off from the Dynkin diagram that the field transforms trivially under the second $S L(2 \mid 1)$ factor, but under the first $S L(2 \mid 1)$ factor it transforms in the representation

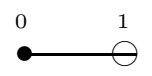

and $Q=1$. This corresponds to a field $W_{A}$ with one downstairs, unprimed superindex, transforming as

$$
W_{A}^{\prime}=\frac{1}{y} W_{B} \tilde{a}_{A}^{B}
$$

which, if we let $W_{A}=\left(W_{\alpha}, W\right)$ becomes

$$
\left(\begin{array}{c}
W_{\alpha}^{\prime} \\
W^{\prime}
\end{array}\right)=\left(\begin{array}{c}
W_{\alpha} / y \\
-W_{\beta} \lambda^{\beta} / y+W
\end{array}\right)
$$

Once again, demanding that both $W_{A}$ and $W_{A}^{\prime}$ are holomorphic in their respective variables leaves the correct components

$$
\begin{aligned}
W_{\alpha} & =\rho_{1 \alpha}+y \rho_{2 \alpha}+\lambda^{\beta} F_{\alpha \beta}-\pi^{\dot{\alpha}} \partial_{\alpha \dot{\alpha}} C-\lambda^{\beta} \pi^{\dot{\beta}} \partial_{\beta \dot{\beta}} \rho_{2 \alpha} \\
W & =C-\lambda^{\alpha} \rho_{2 \alpha}
\end{aligned}
$$

with the components $\left(\rho_{1 \alpha}, \rho_{2 \alpha}, F_{\alpha \beta}, C\right)$ all satisfying their equations of motion.

The conjugate representation is similar. It is usually given as an antichiral field on super Minkowski space and has conjugate Dynkin diagram to (48) on analytic superspace. It is therefore given as a field $W_{A^{\prime}}$ with $Q=1$, transforming as $W_{A^{\prime}} \mapsto W_{A^{\prime}}^{\prime}$ with

$$
W_{A^{\prime}}^{\prime}=\frac{1}{y} d_{A^{\prime}}{ }^{\prime} W_{B^{\prime}}
$$

Its components are the on-shell fields $\left(\sigma_{1 \dot{\alpha}}, \sigma_{2 \dot{\alpha}}, G_{\dot{\alpha} \dot{\beta}}, D\right)$.

As a final example, consider the $N=2$ superconformal stress-energy multiplet. On super Minkowski space it is a scalar superfield $T$ satisfying

$$
D_{\alpha i} D_{j}^{\alpha} T=0 .
$$

It has dilation weight 2 , and all other quantum numbers are 0 . On analytic superspace it has the Dynkin diagram

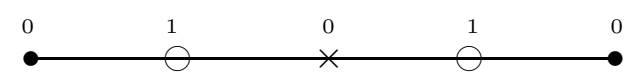

and thus it is given by the superfield $T_{A^{\prime} A}$ with $Q=2$. It thus transforms under $X \mapsto X^{\prime}$ as $T_{A A^{\prime}} \mapsto T_{A A^{\prime}}^{\prime}$ where

$$
T_{A^{\prime} A}^{\prime}=\frac{1}{y^{2}} d_{A^{\prime}}{ }^{\prime} T_{B^{\prime} B} \tilde{a}^{B}{ }_{A} .
$$

Then from demanding that both $T_{A^{\prime} A}$ and $T_{A^{\prime} A}^{\prime}$ are holomorphic in their respective variables leaves us with the correct components of the stress energy multiplet, satisfying the correct equations.

As mentioned previously this representation can also be realised explicitly in two different ways on analytic superspace: firstly by multiplying a Maxwell field and its conjugate together (24) and secondly by multiplying two hypermultiplet fields together with a derivative (25). 


\section{$\mathbf{5 . 5}(4,2,2)$ analytic superspace}

A highest weight representation on $(4,2,2)$ analytic superspace has Dynkin diagram

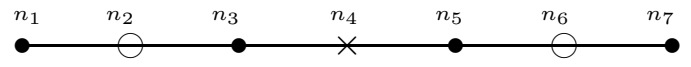

This has Levi subgroup $S(G L(2 \mid 2) \times G L(2 \mid 2))$ and has coordinates $X^{A A^{\prime}}$ given in equation (26). Primed and unprimed capital indices carry representations of the two $G L(2 \mid 2)$ subsupergroups. Using the method of induced representations (254-257), we consider equivariant maps $F: S L(4 \mid 4) \rightarrow V$ where $V$ is the particular representation space for the parabolic subgroup given by the Dynkin diagram. So we have maps such that $F(h u)=R(h) F(u)$ where $u \in S L(4 \mid 4)$ and

$$
h=\left(\begin{array}{ll}
a^{A} B & 0 \\
c_{A^{\prime} B} & d_{A^{\prime}} B^{\prime}
\end{array}\right)
$$

with each entry a $(2 \mid 2)$ matrix. The representation $R$ is given by

$$
R(h)=|a|^{-Q} R^{\left(n_{1}, n_{2}, n_{3}\right)}(a) R^{\left(n_{5}, n_{6}, n_{7}\right)}(d)
$$

where $R^{\left(n_{1}, n_{2}, n_{3}\right)}(a)$ and $R^{\left(n_{5}, n_{6}, n_{7}\right)}(d)$ are tensor representations of $S L(2 \mid 2)$ extended to $G L(2 \mid 2)$ given by the Dynkin labels, $|a|$ is the superdeterminant of $a$ and

$$
Q=-n_{1}+n_{2}+n_{3}+n_{4}+n_{5}+n_{6}-n_{7}=L-J_{1}-J_{2} .
$$

The superalgebra $\mathfrak{s l}(4 \mid 4)$ is not simple, as it contains the identity matrix and thus has a nonzero centre. In order to obtain a simple superalgebra, one has to exclude this to obtain $\mathfrak{p s l}(4 \mid 4)$ which is simple. Representations of the simple super Lie group $P S L(4 \mid 4)$ are representations of $S L(4 \mid 4)$ for which the centre does not act. From (59) we see that the centre is generated by $\hat{R}$ and so representations of $P S L(4 \mid 4)$ are representations of $S L(4 \mid 4)$ with $R=0$. From (61) we obtain

$$
R=\frac{1}{2}\left(n_{1}-2 n_{2}-n_{3}+n_{5}+2 n_{6}-n_{7}\right)
$$

and by considering Young Tableaux one can show that the number of unprimed indices is $n_{3}+2 n_{2}-n_{1}$ and the number of primed indices is $n_{5}+2 n_{6}-n_{7}$ Thus the condition $R=0$ tells us that for representations of $P S L(4 \mid 4)$ the number of primed indices equals the number of unprimed indices.

The only massless multiplet with $R=0$ is the Maxwell multiplet given on $(4,2,2)$ analytic superspace by a field $W$ with charge $Q=1$ and with no superindices. We can obtain other representations of $P S L(4 \mid 4)$ by taking copies of the Maxwell field and applying derivative operators $\partial_{A^{\prime} A}$ to them. For example, consider the representation with internal Dynkin labels $[1,0,1]$, dilation weight $L=2$ and 0 spin. On $(4,2,2)$ space this is given by a field $W_{A^{\prime} A}$ with charge $Q=2$. Since the derivative operator $\partial_{A^{\prime} A}$ has zero charge ${ }^{1}$, we need two copies of the Maxwell field, $W^{(1)}$ and $W^{(2)}$, and one derivative operator to obtain this field. In order for the field to be primary we must take the combination (27) $W_{A^{\prime} A}=W^{(1)} \partial_{A^{\prime} A} W^{(2)}-W^{(2)} \partial_{A^{\prime} A} W^{(1)}$.

\footnotetext{
${ }^{1}$ This is true as long as we only symmetrise the indices as dictated by Young tableau with $m_{1}=0$.
} 
In fact, one can obtain all unitary irreducible representations of $P S L(4 \mid 4)$ (with integer Dynkin labels) in this manner. Given a representation with super Dynkin labels $\left(n_{1}, \ldots, n_{7}\right)$ we need $Q W$ 's and $n_{3}+2 n_{2}-n_{1}=n_{5}+2 n_{6}-n_{7} \partial$ 's. We may need to use higher order derivatives such as $\partial_{A^{\prime} A} \partial_{B^{\prime} B}$, in which case the primed and unprimed super indices will have the same symmetry properties since the $\partial$ 's commute. However it is not hard to convince oneself that for any given representation, we can take a particular combination of $\partial$ 's acting on $W$ 's to obtain that representation. There are many different ways of doing this, we give just one way. We have $Q$ copies of the Maxwell multiplet $W_{i}:(i=1 \ldots Q)$. If $n_{1}<n_{7}$ we can take the following combination

$$
\partial^{n_{1}+2} W_{1} \prod_{i} \partial^{2} W_{i} \prod_{j} \partial W_{j} \prod_{k} W_{k}
$$

where $i, j, k$ are

$$
\begin{aligned}
& n_{3}<n_{5} \Rightarrow\left\{\begin{array}{l}
i=\left(2, \ldots, n_{2}-n_{1}\right) \\
j=\left(n_{2}-n_{1}+1, \ldots, n_{3}+n_{2}-n_{1}\right) \\
k=\left(n_{3}+n_{2}-n_{1}+1, \ldots, Q\right)
\end{array}\right. \\
& n_{5}<n_{3} \Rightarrow\left\{\begin{array}{l}
i=\left(2, \ldots,\left\lfloor n_{6}-\frac{n_{7}+n_{1}}{2}\right\rfloor\right) \\
j=\left(\left\lfloor n_{6}-\frac{n_{7}+n_{1}}{2}\right\rfloor\right) \ldots,\left\lceil n_{5}+n_{6}-\frac{n_{7}+n_{1}}{2}\right\rceil \\
k=\left(\left\lceil n_{5}+n_{6}-\frac{n_{7}+n_{1}}{2}+1\right\rceil, \ldots, Q\right)
\end{array}\right.
\end{aligned}
$$

Here $\lfloor x\rfloor(\lceil x\rceil)$ denote the nearest integers less than (greater than) or equal to $x$. Whenever more than one $\partial$ acts on a $W_{i}$ we take the symmetric combination on both indices. We then project onto the representation of the two $S L(2 \mid 2)$ subgroups with the following Young tableaux
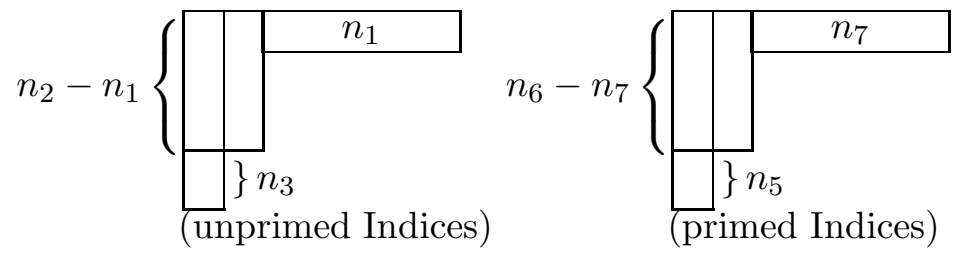

One will have to add other terms in order to make the operator superconformally covariant.

Note that although we can obtain all unitary irreducible representations of $P S L(4 \mid 4)$ with integer Dynkin coefficients in this manner many of the operators of interest - in $N=4$ super Yang-Mills for instance - have anomalous dimensions, which lead to non-integer odd Dynkin coefficients. Such operators can not be given by multiplying together $W$ 's in the above fashion, but they can be represented in analytic superspace as abstract 'quasi-tensor' superfields (section 5.2).

\section{Oscillator method}

Günaydin's oscillator method has been used to obtain the unitary irreducible representations of the conformal group $S U(2,2)$ explicitly [7-11]. These representations can be extended naturally to give representations (no longer unitary) of the complex conformal group $S L(4)$ and one can relate these representations to those acting on fields on complex Minkowski space. In this way we will be able to see that the representations acting on our fields are also unitary. Similarly one can also use the oscillator method to construct unitary representations of the superconformal 
group $S U(2,2 \mid N)$ [7-11]. If one tries to relate these representations to fields in an analogous way to the bosonic case one naturally obtains fields on analytic superspace.

\subsection{The bosonic case}

We here show the oscillator method of obtaining representations of the complex conformal group $S L(4)$ which correspond to unitary representations of the real conformal group $S U(2,2)$, following [9-11]. The lie algebra $\mathfrak{s l}(4)$ is the set of $4 \times 4$ traceless matrices:

$$
\mathfrak{s l}(4)=\left\{\left(\begin{array}{cc}
A^{\alpha}{ }_{\beta} & B^{\alpha \dot{\beta}} \\
C_{\dot{\alpha} \beta} & D_{\dot{\alpha}}^{\dot{\beta}}
\end{array}\right)\right\}
$$

In the following we will refer to the Levi decomposition of $\mathfrak{s l}(4)$ corresponding to Minkowski space, i.e. $\mathfrak{s l}(4)=\mathfrak{n}^{-} \oplus \mathfrak{l} \oplus \mathfrak{n}^{+}$where $\mathfrak{l}$ is the set of block diagonal matrices (matrices in the previous equation such that $B=C=0), \mathfrak{n}^{-}$and $\mathfrak{n}^{+}$are the upper-right and lower-left blocks respectively. One takes $P$ copies of oscillator operators $a^{\alpha}(K)=\left(a_{\alpha}(K)\right)^{\dagger}, b^{\dot{\alpha}}=\left(b_{\dot{\alpha}}\right)^{\dagger}$ transforming under the two $S L(2)$ subgroups corresponding to the matrices $A$ and $D$. Here $K, L=1, \ldots, P$ label different generations of oscillators. The oscillators satisfy the following commutation relations:

$$
\left[a_{\alpha}(K), a^{\beta}(L)\right]=\delta_{\alpha}^{\beta} \delta_{L K} \quad\left[b_{\dot{\alpha}}(K), b^{\dot{\beta}}(L)\right]=\delta_{\dot{\alpha}}^{\dot{\beta}} \delta_{L K}
$$

with all other commutation relations zero. Oscillators with an upper (lower) index are creation (annihilation) operators. The 'vacuum' $|0\rangle$ is defined by

$$
a_{\alpha}|0\rangle=b_{\dot{\alpha}}|0\rangle=0 \text {. }
$$

It is helpful to define 4 -spinors $\psi \underline{\underline{\alpha}}, \bar{\psi}_{\underline{\alpha}}$ as follows

$$
\psi^{\underline{\alpha}}=\left(a^{\alpha}, b_{\dot{\alpha}}\right)^{T} \quad \bar{\psi}_{\underline{\alpha}}=\left(\psi^{\dagger}\right)^{\underline{\beta}} J \underline{\underline{\beta}}=\left(a_{\alpha},-b^{\dot{\alpha}}\right) .
$$

where $J=\operatorname{diag}(1,1,-1,-1)$ so that they satisfy the commutation relation

$$
\left[\psi^{\underline{\beta}}(L), \bar{\psi}_{\underline{\alpha}}(K)\right]=-\delta_{\underline{\alpha}}^{\underline{\beta}} \delta_{K L} .
$$

If we call the matrix in (172) $M^{\underline{\alpha}} \underline{\beta}$ and define

$$
\widehat{M}:=-\sum_{K=1}^{P} \bar{\psi}_{\underline{\alpha}}(K) M_{\underline{\underline{\alpha}}}^{\underline{\alpha}} \psi \underline{\underline{\beta}}(K)
$$

we find

$$
\left[\widehat{M}, \widehat{M^{\prime}}\right]=\left[\widehat{M, M^{\prime}}\right]
$$

So $\widehat{M}$ is an infinite dimensional representation of $\mathfrak{s l}(4)$ acting on the Fock space of the oscillators. The dilation weight $L$ is generated by

$$
\widehat{L}=\frac{1}{2}\left(a_{\alpha} \cdot a^{\alpha}+b^{\dot{\alpha}} \cdot b_{\dot{\alpha}}\right)=\frac{1}{2}\left(N_{a}+N_{b}+2 P\right)
$$


where the dot corresponds to summation over the $P$ different oscillator generations, and where $N_{a}=a^{\alpha} \cdot a_{\alpha}, N_{b}=b^{\dot{\alpha}} \cdot b_{\dot{\alpha}}$ are the bosonic number operators. The irreducible representations of $\mathfrak{s l}(4)$ are obtained by constructing an irreducible representation, $\langle\Omega|$, of the parabolic subalgebra $\mathfrak{l} \oplus \mathfrak{n}^{+}$in the Fock space of the oscillators. As mentioned in the appendix, irreducible representations of parabolic subgroups are acted on trivially by the nilpotent part $\mathfrak{n}^{+}$, therefore we insist that $\langle\Omega|$ is annihilated by the generators which correspond to the generators $\mathfrak{n}^{+}$

$$
\langle\Omega| a^{\alpha} \cdot b^{\dot{\alpha}}=0
$$

Applying the operators $a_{\alpha} \cdot b_{\dot{\alpha}}$ (which correspond to $\mathfrak{n}^{-}$) repeatedly on $\langle\Omega|$ one generates the infinite set of states

$$
\langle\Omega|, \quad\langle\Omega| a_{\alpha} \cdot b_{\dot{\alpha}}, \quad\langle\Omega| a_{\alpha} \cdot b_{\dot{\alpha}} a_{\beta} \cdot b_{\dot{\beta}}, \quad \ldots
$$

which form a basis for the irreducible representation of $S L(4)$. Similarly a set of states are obtained from the conjugate representation $|\Omega\rangle$, which is annihilated by $a_{\alpha} \cdot b_{\dot{\alpha}}$

$$
|\Omega\rangle, \quad a^{\alpha} \cdot b^{\dot{\alpha}}|\Omega\rangle, \quad a^{\alpha} \cdot a^{\dot{\alpha}} a^{\beta} \cdot b^{\dot{\beta}}|\Omega\rangle, \quad \ldots
$$

The equivalence between this formulation of the representations, and field representations on complex Minkowski space is straightforward. The state $|\Phi\rangle$ corresponds to the field $\Phi(x)$ given by

$$
\Phi\left(x^{\alpha \dot{\alpha}}\right)=\langle\Omega|\widehat{s}(x)| \Phi\rangle
$$

where $\widehat{s}(x)=\exp \left(x^{\alpha \dot{\alpha}} a_{\alpha} \cdot b_{\dot{\alpha}}\right)$ corresponds to the coset representative of $\mathfrak{s l}(4)$ for complex Minkowski space (259). The field $\Phi(x)$ therefore inherits the index structure of $\langle\Omega|$. Under an $\mathfrak{s l}(4)$ transformation $|\Phi\rangle \mapsto \widehat{M}|\Phi\rangle$ the field transforms as

$$
\Phi(x) \mapsto\langle\Omega|\widehat{s}(x) \widehat{M}| \Phi\rangle=\left\langle\Omega\left|\widehat{h}(x, g) \widehat{s}\left(x^{\prime}\right)\right| \Phi\right\rangle=R(h(x, g)) \Phi\left(x^{\prime}\right)
$$

exactly as an induced representation should (257). Explicitly the correspondence can be written in terms of the Taylor series for $\Phi(x)$ as

$$
|\Phi\rangle=\Phi(0)|\Omega\rangle+\partial_{\alpha \dot{\alpha}} \Phi(0) a^{\alpha} \cdot b^{\dot{\alpha}}|\Omega\rangle+\ldots
$$

For example, if we take $P=1$ so there is only one generation of oscillators, then the only possible forms $|\Omega\rangle$ can have are

$$
\langle 0| a_{\alpha_{1}} \ldots a_{\alpha_{2 J_{1}}}
$$

and

$$
\langle 0| b_{\dot{\alpha}_{1}} \ldots b_{\dot{\alpha}_{2 J_{2}}} \text {. }
$$

Using the expressions for the dilation weight (179) and (261) we find that these have Dynkin coefficients

$$
\stackrel{2 J_{1}-\left(2 J_{1}+1\right) \quad 0}{\longleftarrow} \bullet
$$

These correspond to massless fields on Minkowski space. For example, take $\langle\Omega|=\langle 0|$ then we obtain the representation corresponding to a massless scalar. Using the relation (183) notice that

$$
\partial_{\alpha \dot{\alpha}} \partial_{\beta \dot{\beta}} \Phi(x)=\left\langle 0\left|a_{\alpha} b_{\dot{\alpha}} a_{\beta} b_{\dot{\beta}} \widehat{s}(x)\right| \Phi\right\rangle
$$


and therefore since there is only one generation of oscillators, this is symmetric in the indices $\alpha, \beta$, and thus $\Phi$ satisfies the wave equation

$$
\partial_{\dot{\alpha}[\alpha} \partial_{\beta] \dot{\beta}} \Phi=0 \Longrightarrow \square \Phi=0
$$

If we take $\langle\Omega|=\langle 0| a_{\alpha}$ then we obtain a chiral spinor field. Again (183) gives

$$
\partial_{\alpha \dot{\alpha}} \Psi_{\beta}=\left\langle 0\left|a_{\beta} a_{\alpha} b_{\dot{\alpha}} \widehat{s}(x)\right| \Psi\right\rangle
$$

and this is also symmetric in the indices $\alpha, \beta$ and so $\Psi(x)$ must satisfy the spinor equation

$$
\partial_{\dot{\alpha}[\alpha} \Psi_{\beta]}=0
$$

\subsection{Unitarity in the bosonic case}

So far the discussion has simply been about constructing representations of the complexified conformal group $S L(4)$. We now wish to look at the restriction of these representations to representations of the real superconformal group $S U(2,2)$. There are two ways of restricting the Lie algebra $\mathfrak{s l}(4)$ to $\mathfrak{s u}(2,2)$. The first choice is known as the compact basis, since in this basis the Levi subgroup of $S L(4)$ becomes the compact group $S U(2) \times S U(2) \times U(1)$. This is the choice usually associated with the oscillator method [9-11]. It is obtained by restricting our $\mathfrak{s l}(4)$ matrices as follows

$$
M J+J M^{\dagger}=0
$$

where $J=\operatorname{diag}(1,1,-1,-1)$. Note that if $M$ satisfies this condition then from (175)

$$
\widehat{M}^{\dagger}+\widehat{M}=0 \text {. }
$$

So the representation of $S U(2,2)$ obtained by exponentiating $\widehat{M}$ is unitary with the natural inner product

$$
\left(\phi, \phi^{\prime}\right)=\left\langle\phi \mid \phi^{\prime}\right\rangle
$$

However, we are interested in the choice of $S U(2,2)$, known as the non-compact basis. This is defined by the restriction

$$
M^{\prime} K+K M^{\prime \dagger}=0
$$

where

$$
K=\left(\begin{array}{ll}
0_{2} & 1_{2} \\
1_{2} & 0_{2}
\end{array}\right) .
$$

It is this basis for which the induced representations act on fields defined on real Minkowski space.

The two choices for the group $S U(2,2)$ are related by the following

$$
M=U^{-1} M^{\prime} U
$$

where

$$
U=\frac{1}{\sqrt{2}}\left(\begin{array}{cc}
1_{2} & 1_{2} \\
-1_{2} & 1_{2}
\end{array}\right)
$$


Since $U$ is an element of the group $S L(4)$, there is a corresponding element $\widehat{U}$ in the Fock space of the oscillators and we obtain the analogue of equation (198)

$$
\widehat{M}=\widehat{U}^{-1} \widehat{M^{\prime}} \widehat{U}
$$

Then (194) gives

$$
\widehat{V} \widehat{M}^{\prime}+\widehat{M}^{\prime} \widehat{V}^{\prime}=0
$$

where $\widehat{V}=\left(\widehat{U} \widehat{U}^{\dagger}\right)^{-1}$. So in this basis the representations are still unitary, but with an unusual inner product

$$
\left(\phi, \phi^{\prime}\right)=\left\langle\phi|\widehat{V}| \phi^{\prime}\right\rangle
$$

\subsection{The supersymmetric case}

The oscillator construction of the previous section can be generalised to construct unitary irreducible representations of the superconformal group $S U(2,2 \mid N)$ [7-11]. Again we will complexify this and use it to construct the corresponding irreducible representations of the complex superconformal group $S L(4 \mid N)$. We will show the relationship between these and field representations on analytic superspace in analogy with the above relationship to fields on Minkowski space. For work on the relationship between oscillator representations and fields on super Minkowski space in six dimensions see [41].

We consider the decomposition of the Lie algebra $\mathfrak{s l}(4 \mid N)$ corresponding to $(N, p, N-p)$ analytic space, which has Levi subgroup $S L(2 \mid p) \oplus S L(2 \mid N-p) \oplus \mathbb{C}$. The Lie algebra $\mathfrak{s l}(4 \mid N)$ consists of matrices $M$ of the following form

$$
M=\left(\begin{array}{cc}
A_{B}^{A} & B^{A B^{\prime}} \\
C_{A^{\prime} B} & D_{A^{\prime}} B^{\prime}
\end{array}\right) .
$$

where the index $A=(\alpha, a)$ and $A^{\prime}=\left(a^{\prime}, \dot{\alpha}\right)$ and $a=1 \ldots p, a^{\prime}=p+1, \ldots N$ we can then define generalised oscillators $\xi_{A}, \xi^{A}=\xi_{A}^{\dagger}$ and $\eta_{A^{\prime}}, \eta^{A^{\prime}}=\eta_{A}^{\dagger}$ transforming under the supergroups $S L(2 \mid p)$ and $S L(2 \mid N-p)$ respectively. The oscillators $\xi^{a}, \xi_{a}, \eta^{a^{\prime}}, \eta_{a^{\prime}}$ are odd and all others are even. They satisfy the following generalised commutation relations

$$
\left[\xi_{A}(K), \xi^{B}(L)\right]=\delta_{A}^{B} \delta_{L K} \quad\left[\eta_{A^{\prime}}(K), \eta^{B^{\prime}}(L)\right]=\delta_{A^{\prime}}^{B^{\prime}} \delta_{L K}
$$

where these are commutation relations unless both oscillators are odd in which case they are anticommutation relations with annihilation (creation) operators labelled by lower (upper) indices.

The Levi decomposition is $\mathfrak{s l}(4 \mid N)=\mathfrak{n}^{-} \oplus \mathfrak{l} \oplus \mathfrak{n}^{+}$where the Levi subgroup corresponds to the block diagonal matrices with $B=C=0, \mathfrak{n}^{-}$and $\mathfrak{n}^{+}$correspond to the upper right and lower left blocks respectively. The 'vacuum' is defined by

$$
\xi_{A}|0\rangle=\eta_{A^{\prime}}|0\rangle=0
$$

We define the objects $\Psi \underline{A}=\left(\xi^{A}, \eta_{A^{\prime}}\right)^{T}$ and $\bar{\Psi}_{\underline{A}}=\Psi_{\underline{B}}^{\dagger} J \underline{\underline{B}}=\left(\xi_{\alpha},-\xi_{a},-\eta^{a^{\prime}},-\eta^{\dot{\alpha}}\right)$ where $J=$ $\operatorname{diag}\left(1_{2},-1_{p},-1_{N-p},-1_{2}\right)$. These satisfy the generalised commutation relation

$$
\left[\Psi^{\underline{A}}, \bar{\Psi}_{\underline{B}}\right]=-\delta \underline{A} .
$$


For $M \in \mathfrak{s l}(4 \mid N)$ we define

$$
\widehat{M}:=-\sum_{K=1}^{P} \bar{\Psi}_{\underline{A}}(K) M \underline{A}_{\underline{B}} \Psi \underline{B}(K)
$$

and find that

$$
\left[\widehat{M}, \widehat{M}^{\prime}\right]=\left[\widehat{M, M^{\prime}}\right]
$$

So again $\widehat{M}$ is an infinite dimensional representation of $\mathfrak{s l}(4 \mid N)$ acting on the Fock space of the oscillators.

The charge $Q$ for $(N, p, N-p)$ analytic superspace (defined as in (143)) turns out to be equal to the number of generations of oscillators

$$
Q=P
$$

One obtains the irreducible representations by constructing an irreducible representation, $\langle\Omega|$, of the parabolic subalgebra $\mathfrak{l} \oplus \mathfrak{n}^{+}$which will in fact be annihilated by $\mathfrak{n}^{+}$. The conjugate representation $|\Omega\rangle$ carries an irreducible representation of the parabolic subgroup $\mathfrak{l} \oplus \mathfrak{n}^{-}$which is annihilated by $\mathfrak{n}^{-}$. A basis for this representation is obtained by acting on $|\Omega\rangle$ with successive elements of $\mathfrak{n}^{+}$

$$
|\Omega\rangle, \quad \xi^{A} \cdot \eta^{A^{\prime}}|\Omega\rangle, \quad \xi^{A} \cdot \eta^{A^{\prime}} \xi^{B} \cdot \eta^{B^{\prime}}|\Omega\rangle, \ldots
$$

We can then make explicit the relationship between these representations and fields on $(N, p, q)$ analytic superspace as in the bosonic case. A state $|\Phi\rangle$ corresponds to the field $\Phi(X)$ where

$$
\Phi(X)=\langle\Omega|\widehat{s}(X)| \Phi\rangle
$$

and where $\widehat{s}(X)=\exp \left(\xi_{A}(K) X^{A A^{\prime}} \eta_{A^{\prime}}(K)\right)$ (with the $K$ 's summed over) corresponds to the coset representative of $\mathfrak{s l}(4 \mid N)$ for $(N, p, N-p)$ analytic superspace (as in for example (134)). Just as in the bosonic case one can check that under the $\mathfrak{s l}(4 \mid N)$ transformation $|\Phi\rangle \mapsto \widehat{M}|\Phi\rangle$ the field defined thus transforms as a field on analytic space. ${ }^{2}$ Explicitly

$$
|\Phi\rangle=\Phi(0)|\Omega\rangle+\eta^{A^{\prime}} \partial_{A^{\prime} A} \xi^{A} \Phi(0)|\Omega\rangle+\ldots
$$

For example, if we take $P=1$ then the possible forms $\langle\Omega|$ can have are

$$
\langle\Omega|=\langle\Omega| \xi_{A_{1}} \xi_{A_{2}} \ldots \xi_{A_{s}}
$$

and

$$
\langle\Omega|=\langle\Omega| \eta_{A_{1}^{\prime}} \eta_{A_{2}^{\prime}} \ldots \eta_{A_{s}^{\prime}} .
$$

So for $(2,1,1)$ analytic superspace consider the case $\langle\Omega|=\langle 0|$. According to (211) this gives rise to a superfield with no superindices, and a charge $Q=1$, i.e. the field $W$ on analytic space corresponding to the hypermultiplet (153). The case $\langle\Omega|=\langle 0| \xi_{A}$ also has one (undashed) superindex and $Q=1$. It therefore gives rise to the field $W_{A}$ on analytic superspace corresponding to the Maxwell multiplet (157).

\footnotetext{
${ }^{2}$ Note that there is a small difference with the field transformations here and the choice defined in (151): here the combinations $W_{A} V^{A}$ and $W^{A^{\prime}} V_{A^{\prime}}$ transform as scalars, whereas with our previous choice $W^{A} V_{A}$ and $W^{A^{\prime}} V_{A^{\prime}}$ were scalars.
} 
If we allow two generations of oscillators $P=2$ then we can have

$$
\langle\Omega|=\langle 0| \eta_{A^{\prime}}(2) \xi_{A}(1)
$$

with charge $Q=2$. This gives the field $T_{A^{\prime} A}$ transforming as in (163) and corresponding to the stress-energy multiplet.

\subsection{Unitarity in the supersymmetric case}

There are two different forms for the real superconformal group $S U(2,2 \mid N)$ as there were for the conformal group. The natural form for the oscillator construction, and the one taken in [9-11] is the compact basis where the Lie algebra elements $M \in \mathfrak{s l}(2,2 \mid N)$ satisfy $M J+J M^{\dagger}=0$ where $J=\operatorname{diag}\left(1_{2},-1_{p},-1_{N-p},-1_{2}\right)$. In this case, from the definitions of $\widehat{M}$ and $\bar{\Psi}$ we find that

$$
\widehat{M}+\widehat{M}^{\dagger}=0
$$

and so the oscillator representations are unitary with the natural inner product

$$
\left(\Phi, \Phi^{\prime}\right)=\left\langle\Phi \mid \Phi^{\prime}\right\rangle
$$

We are interested in the non-compact basis of $S U(2,2 \mid N)$, which leads to fields on real spacetime. This is defined by restricting $M^{\prime} \in \mathfrak{s l}(4)$ to satisfy $M^{\prime} K+K M^{\prime \dagger}=0$ where

$$
K=\left(\begin{array}{l|l|l} 
& & 1_{2} \\
\hline & 1_{N} & \\
\hline 1_{2} & &
\end{array}\right) .
$$

These two forms of the Lie algebra $\mathfrak{s l}(4 \mid N)$ are related to each other by $M=U^{-1} M^{\prime} U$ where

$$
U=\left(\begin{array}{c|c|c}
1 / \sqrt{2} & & 1 / \sqrt{2} \\
\hline & 1 & \\
\hline-1 / \sqrt{2} & & 1 / \sqrt{2}
\end{array}\right) .
$$

Since $U \in S L(4 \mid N)$ there is a corresponding element $\widehat{U}$ in our representation space, and (216) implies that

$$
\widehat{V} \widehat{M^{\prime}}+M^{\prime \dagger} \widehat{V}=0
$$

where $\widehat{V}=\left(\widehat{U} \widehat{U}^{\dagger}\right)^{-1}$. So representations are unitary with respect to the inner product

$$
\left(\Phi, \Phi^{\prime}\right)=\left\langle\Phi|\widehat{V}| \Phi^{\prime}\right\rangle
$$

\section{Acknowledgements}

I am very grateful to my supervisor P.S.Howe for suggestions, criticism and helpful conversations. This research was supported by a PPARC studentship and PPARC SPG grant 613.

\section{A Appendix}

We here set out some of the general formalism in the purely bosonic case which we have applied in the supersymmetric case. 


\section{A.1 Roots and parabolic subalgebras}

Let $\mathfrak{g}$ be a complex semisimple Lie algebra and $G$ the corresponding Lie group. We fix a Cartan subalgebra $\mathfrak{h}$ of $\mathfrak{g}$ and for $\alpha \in \mathfrak{h}^{*}$ the dual space of $\mathfrak{h}$ we define

$$
\mathfrak{g}_{\alpha}=\{v \in \mathfrak{g}:[v, h]=\alpha(h) v\} .
$$

Then the collection

$$
\Delta=\left\{\alpha \in \mathfrak{h}^{*}: \mathfrak{g}_{\alpha} \neq 0, \alpha \neq 0\right\}
$$

is called the set of roots of $\mathfrak{g}$ relative to $\mathfrak{h}$. Now from the Jacobi identity $\left[\mathfrak{g}_{\alpha}, \mathfrak{g}_{\beta}\right] \subset \mathfrak{g}_{\alpha+\beta}$ and so the roots span an integral lattice in $\mathfrak{h}^{*}$. We choose a system of simple roots of $\mathfrak{g} S=\left\{\alpha_{i}\right\}$ such that any $\alpha \in \Delta$ can be expressed as a linear combination of elements of $S$ with either all non-negative coefficients, or all non-positive coefficients. We can choose a different system of simple roots, but all choices are equivalent up to conjugation $\left(g \mathfrak{g} g^{-1}\right.$ where $g \in G$ is defined to be a conjugate Lie algebra.) Once we have defined $S$ we define the positive roots $\Delta^{+}$as

$$
\Delta^{+}=\left\{\alpha \in \Delta: \alpha=\sum a_{i} \alpha_{i}, \quad a_{i} \geq 0, \quad \alpha_{i} \in S\right\} .
$$

Now $\mathfrak{g}$ admits a bilinear form known as the Killing form which, for $u, v \in \mathfrak{g}$, is

$$
(u, v)=\operatorname{tr} \operatorname{ad}(u) \operatorname{ad}(v)
$$

where ad is the adjoint representation of $\mathfrak{g}$. This form is non-degenerate on $\mathfrak{h}$ and we can thus identify $\mathfrak{h}^{*}$ with $\mathfrak{h}$ by means of this form: identify $h_{\alpha} \in \mathfrak{h}$ with $\alpha \in \mathfrak{h}^{*}$ where

$$
\alpha(h)=\left(h, h_{\alpha}\right) \forall h \in \mathfrak{h} .
$$

We can then define a bilinear form on $\mathfrak{h}^{*}$ by

$$
(\alpha, \beta):=\left(h_{\alpha}, h_{\beta}\right)
$$

For each root $\alpha$ we define its co-root $\alpha^{\vee}$ as

$$
\alpha^{\vee}=2 \alpha /(\alpha, \alpha)
$$

This always exists as it can be shown that for all roots $\alpha,(\alpha, \alpha)>0$. We will define $H_{i} \in \mathfrak{h}$ to be the Cartan subalgebra elements associated with the simple co-roots

$$
H_{i}=h_{\alpha_{i}^{\vee}}
$$

From this we can define the Cartan Matrix

$$
c_{i j}=\left(\alpha_{i}, \alpha_{j}^{\vee}\right)=\alpha_{i}\left(H_{j}\right)
$$

where $\alpha_{i} \in S$ which uniquely specifies $\mathfrak{g}$. A useful way of describing the Cartan Matrix is with a Dynkin diagram. This is a graph with nodes corresponding to the simple roots $\alpha_{i}$ and with $L_{j k}=\max \left|c_{j k}\right|,\left|c_{k j}\right|$ edges connecting node $j$ to node $k$. 
For the Lie algebra $\mathfrak{g}=\mathfrak{s l}(n)$ the Cartan subalgebra is the set of diagonal matrices (with unit determinant). The roots are $e_{i j} \in \mathfrak{h}^{*}$ where $1 \leq i \neq j \leq n$ given by

$$
e_{i j}(h)=h_{i}-h_{j}
$$

where $\mathfrak{h} \ni h=\operatorname{diag}\left(h_{1}, h_{2}, \ldots h_{n}\right)$ and the corresponding subspaces $\mathfrak{g}_{e_{i j}}$ are spanned by the matrices $\hat{e}_{j i} \in \mathfrak{s l}(N)$ with zeros everywhere except in the $j i$ component where there is a 1 . Simple roots can be chosen as

$$
\alpha_{i}=\left\{e_{i(i+1)}: i=1 \ldots n-1\right\} .
$$

In this case the bilinear form (225) simplifies to

$$
(u, v)=2 N \operatorname{tr}(u v)
$$

The simple co-roots are $\alpha_{i}^{\vee}=2 N \alpha_{i}$ and the corresponding member of the Cartan subalgebra is

$$
H_{i}=\hat{e}_{i i}-\hat{e}_{(i+1)(i+1)} \text {. }
$$

One can then work out the Cartan matrix and the Dynkin diagram. The Dynkin diagram for $\mathfrak{s l}(N)$ is given by $N-1$ nodes each connected by single edges to the adjacent nodes

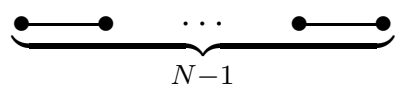

The standard Borel subalgebra $\mathfrak{b}$ is given by

$$
\mathfrak{b}=\mathfrak{h} \oplus \mathfrak{n}
$$

where

$$
\mathfrak{n}=\bigoplus_{\alpha \in \Delta^{+}} \mathfrak{g}_{\alpha}
$$

A parabolic subalgebra $\mathfrak{p}$ is defined to be one which contains the Borel subalgebra. Alternatively specify a subset $S_{\mathfrak{p}}$ of $S$ and define

$$
\Delta(l):=\operatorname{Span} S_{\mathfrak{p}} \cap \Delta
$$

where Span $S_{\mathfrak{p}}$ is the subspace of $\mathfrak{h}^{*}$ spanned by linear combinations of the elements of the root vectors in $S_{\mathfrak{p}}$. Then define

$$
\mathfrak{l}:=\mathfrak{h} \oplus \bigoplus_{\alpha \in \Delta(\mathfrak{l})} \mathfrak{g}_{\alpha}
$$

and

$$
\mathfrak{n}=\bigoplus_{\alpha \in \Delta(\mathfrak{n})} \mathfrak{g}_{\alpha}
$$

where $\Delta(\mathfrak{n})=\Delta^{+} \backslash \Delta(\mathfrak{l})$. The subalgebra defined as

$$
\mathfrak{p}:=\mathfrak{l} \oplus \mathfrak{n}
$$


contains the Borel subalgebra and is thus parabolic, and up to conjugation, all parabolics arise in this way (i.e. all parabolics are of the form $g^{-1} \mathfrak{p} g$ for some $g \in G$ and some standard parabolic $\mathfrak{p}$ constructed as above.) The subgroup $\mathfrak{l}$ is known as the Levi subgroup.

For $\mathfrak{s l}(N)$ the standard Borel subalgebra is given by the set of traceless lower triangular matrices, and the standard parabolic subalgebras are the set of traceless 'block lower-triangular' matrices:

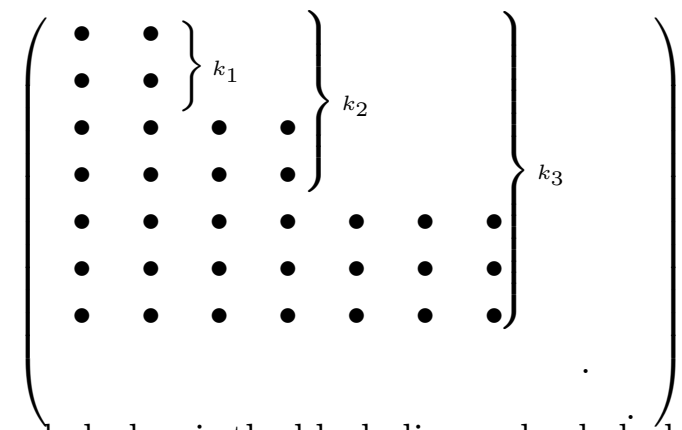

The corresponding Levi subalgebra is the block diagonal subalgebra, i.e. $\mathfrak{s}\left(\mathfrak{g l}\left(k_{1}\right) \oplus \mathfrak{g l}\left(k_{2}-k_{1}\right) \oplus\right.$ ...).

A useful notation for a standard parabolic $\mathfrak{p} \subseteq \mathfrak{g}$ is to cross through all the nodes in the Dynkin diagram corresponding to the simple roots in $S \backslash S_{\mathfrak{p}}$. So the parabolic in our example (242) would be represented by placing a cross on each of the nodes $k_{1}, k_{2}, \ldots, k_{l}$ of the Dynkin diagram for $\mathfrak{s l}(N)$. The Borel subalgebra is represented by a Dynkin diagram with all nodes crossed out.

\section{A.2 Representations}

If $W$ is a representation space for $\mathfrak{g}$ then $0 \neq w \in W$ is a weight vector of weight $\lambda \in \mathfrak{h}^{*}$ if and only if

$$
R(h) w=\lambda(h) w \quad \forall h \in \mathfrak{h}
$$

where $R$ is the representation. We define raising and lowering subalgebras of $\mathfrak{g}$ respectively as

$$
\mathfrak{n}=\bigoplus_{\alpha \in \Delta^{+}} \mathfrak{g}_{\alpha} ; \quad \mathfrak{n}_{-}=\bigoplus_{\alpha \in \Delta^{-}} \mathfrak{g}_{\alpha}
$$

(For $\mathfrak{s l}(N), \mathfrak{n}$ is the set of lower triangular matrices excluding the diagonals, and $\mathfrak{n}_{-}$is the set of upper triangular matrices excluding the diagonals.) Note that if $w$ is a weight vector of weight $\lambda$, and $v \in \mathfrak{g}_{\alpha}$ then $\left.(243,222)\right)$ give

$$
R(h) R(v) w=(\lambda-\alpha)(h) R(v) w
$$

and so $R(v) w$ is a weight vector with weight $\lambda-\alpha$. We define a partial ordering on the root space by

$$
\lambda \preceq \lambda^{\prime} \Leftrightarrow \lambda=\lambda^{\prime}+a_{i} \alpha_{i}, \quad a_{i} \geq 0, \quad \alpha_{i} \in \Delta^{+} .
$$

So applying lowering operators on a weight space of weight $\lambda$ produces a weight space with weight $\lambda^{\prime} \preceq \lambda$. A maximal weight state $w \in W$ with weight $\lambda$ is annihilated by all raising operators and a maximal weight state is a highest weight state if $\lambda \succeq \lambda^{\prime} \forall \lambda^{\prime} \in \Delta$. A set of weights $\lambda_{i}$ dual to the simple roots are defined by

$$
\left(\lambda_{i}, \alpha_{j}^{\vee}\right)=\delta_{i j}
$$


Then any weight $\lambda$ is expressed uniquely as

$$
\lambda=\sum_{i}\left(\lambda, \alpha_{i}^{\vee}\right) \lambda_{i}
$$

We can then represent a weight $\lambda$ by putting the coefficient

$$
n_{j}=\left(\lambda, \alpha_{j}^{\vee}\right)=\lambda\left(H_{j}\right)
$$

over the node of the Dynkin diagram for $\mathfrak{g}$ which corresponds to the root $\alpha_{j}$. A weight $\lambda$ is said to be integral if all the coefficients are integers and dominant if they are all non-negative.

Now it turns out that any finite dimensional representation of $\mathfrak{g}$ has a unique highest weight vector which is dominant integral and there is a one-to-one correspondence between dominant integral highest weight states and finite dimensional irreducible representations. We will however be mostly interested in infinite dimensional representations, which still have a unique highest weight state. These will be called highest weight representations. We will thus use the Dynkin diagram with Dynkin coefficients, both to describe the weight, and also the irreducible representation which has this weight as its highest weight.

A similar statement can be made for representations of a parabolic subalgebra $\mathfrak{p}$. In fact, if $\mathfrak{p}=\mathfrak{l} \oplus \mathfrak{n}$ is a Levi decomposition of $\mathfrak{p}$ then $\mathfrak{n}$ acts trivially on any irreducible representation of $\mathfrak{p}$ and so an irreducible representation of $\mathfrak{p}$ corresponds to an irreducible representation of the Levi subalgebra $\mathfrak{l}$. $\mathfrak{l}$ splits into a semisimple part and a centre

$$
\mathfrak{l}=\mathfrak{l}_{S}+\mathfrak{l}_{Z} \text { where } \mathfrak{l}_{S}=[\mathfrak{l}, \mathfrak{l}]
$$

So an irreducible representation of $\mathfrak{p}$ corresponds to an irreducible representation of $\mathfrak{l}_{S}$ together with $\mathbb{C}$-charges giving a representation of $\mathfrak{l}_{Z}$ and we know that the representation of $\mathfrak{l}_{S}$ has a unique highest weight from above. A weight $\lambda \in \mathfrak{h}^{*}$ is said to be integral or dominant for $\mathfrak{p}$ if $\tau(\lambda)$ is dominant or integral for $\mathfrak{l}_{S}$ where $\tau: \mathfrak{h}^{*} \rightarrow(\mathfrak{h} \cap \mathfrak{l})^{*}$ is the natural projection operator. It is then clear that finite dimensional representations of $\mathfrak{p}$ are in one-to-one correspondence with weights which are dominant integral for $\mathfrak{p}$. Diagrammatically this is represented by insisting that all the coefficients corresponding to nodes which are not crossed through in the Dynkin diagram must be non-negative integers, i.e. $\left(\lambda, \alpha_{i}^{\vee}\right) \in \mathbb{Z}^{+} \cup\{0\}$ for all $\alpha_{i} \in S_{\mathfrak{p}}$. Furthermore, in order for the representation to exponentiate to a representation of the Lie group $P$, the highest weight must be integral (but not necessarily dominant) for $\mathfrak{g}$ and not just for $\mathfrak{p}$. In other words finite dimensional irreducible representations of $P$ correspond to Dynkin diagrams with crosses through some nodes and integral coefficients above the nodes, where the integers above nodes which are not crossed out must be non-negative.

\section{A.3 Tensor representations}

All finite dimensional irreducible representations of $\mathfrak{s l}(n)$ can be given explicitly as tensor products of the fundamental representation. These tensor products are symmetrised to put them into irreducible representations. The symmetry properties of these tensor products is given using 
Young Tableaux in the usual way.

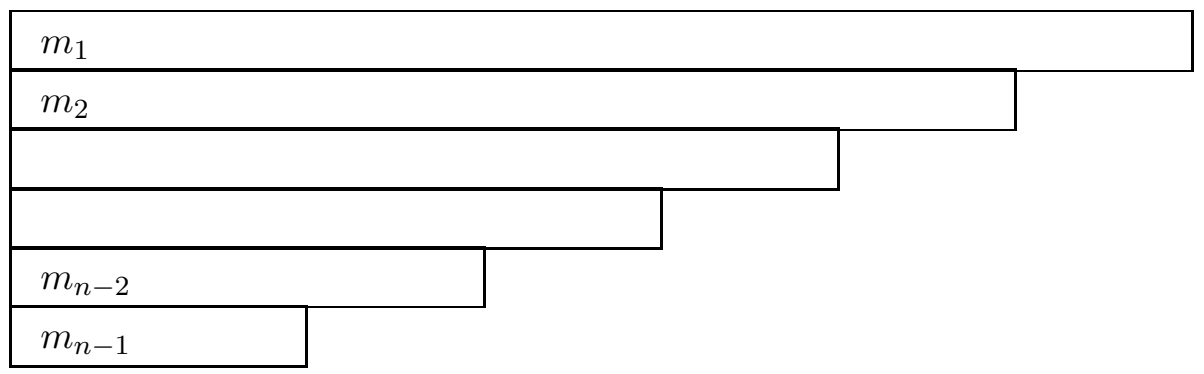

where there are $m_{i}$ squares in each row. To convert from a Young Tableau notation to a Dynkin diagram for a particular representation one we must find the highest weight state of the tensor representation. For $\mathfrak{s l}(n)$ this is obtained by putting 1's in the first row of the Young Tableau, 2 's in the second row, and so on. From $(243,249)$ we see that

$$
R\left(H_{i}\right) w=n_{i} w
$$

if $w$ is the highest weight state. Applying this formula and our expression for $H_{i}(234)$ we obtain

$$
n_{i}=m_{i}-m_{i+1}
$$

\section{A.4 Induced representations}

One way of giving representations of a group is with induced representations which we now describe. If $G$ is a Lie group and $P$ is a subgroup of $G$ we form the coset space $X$ of right cosets $X=P \backslash G$. Fields on these spaces are equivariant maps $F: G \rightarrow V$, where $V$ is a representation space for $P$, i.e. maps such that

$$
F(h u)=R(h) F(u)
$$

where $u \in G, h \in P$ and $R$ is the representation of $P$ on $V$. These fields form representations of $G$ as follows:

$$
(g \cdot F)(u)=F(u g)
$$

In practice one uses local sections $s: U \rightarrow G$ of the bundle $G \rightarrow H \backslash G, U \ni x \mapsto s(x)$ where $U$ is an open subset of $X$. Under a group transformation $g \in G, x \mapsto x^{\prime}$ where $x^{\prime}$ can be found by the following formula:

$$
s(x) g=h(x, g) s\left(x^{\prime}\right)
$$

$h \in P$. Thus

$$
(g \cdot F)(s(x))=R(h(x, g)) F\left(s\left(x^{\prime}\right)\right) .
$$

The method of parabolic induction involves induced representations of a complex, simple Lie group $G$ with a standard parabolic subgroup $P$, the maps $F$ being holomorphic on $P \backslash G$. In this case the coset space $X=P \backslash G$ is known as a flag manifold. For example complexified, compactified Minkowski space can be viewed as the coset space $P \backslash S L(4), S L(4)$ being the 
complexified conformal group and $P$ the parabolic subgroup of matrices of the following shape:

$$
\left(\begin{array}{llll}
\bullet & \bullet & & \\
\bullet & \bullet & & \\
\bullet & \bullet & \bullet & \bullet \\
\bullet & \bullet & \bullet & \bullet
\end{array}\right)
$$

where the bullets denote elements which do not have to be zero. The blank region can be thought of as corresponding to spacetime. Indeed, it takes six charts to cover the whole of compactified complexified Minkowski space. We focus on the coset representative of the form

$$
X \ni x \mapsto s(x)=\left(\begin{array}{cc}
1_{2} & x \\
0_{2} & 1_{2}
\end{array}\right)
$$

which can be identified with non-compact (affine) Minkowski space. Here each entry is a twoby-two matrix and $x$ is a matrix representation of the coordinates for complexified Minkowski space. The transformation of $x$ under the conformal group can easily be calculated using the above formalism, and agrees with the usual one. Here the Levi subalgebra is $\mathfrak{s}(\mathfrak{g l} l(2) \oplus \mathfrak{g l}(2))$ and the Dynkin diagram is $\bullet \bullet \bullet$.

To specify an induced representation on a flag manifold $P \backslash G$ we need to give the representation $R$ of the parabolic subalgebra $P$ under which our fields transform. But the finite dimensional irreducible representations of $P$ have been described above. They can be represented by a Dynkin diagram with crosses through some nodes and integer coefficients above each node, the integers above uncrossed nodes being non-negative. We use this same Dynkin diagram to describe the induced representation.

The Borel-Weil theorem states that if $\lambda \in \mathfrak{h}^{*}$ is dominant integral for $G$ then for any parabolic subgroup $P$ the induced representation specified by this $\lambda$ is isomorphic to the unique finite dimensional irreducible representation specified by the highest weight $\lambda$. So diagrammatically, if $a_{i} \geq 0$ for all $i$ then

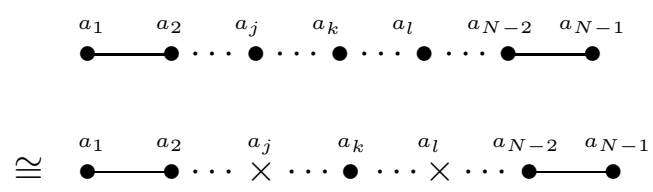

where crosses can be placed on any of the nodes of the right hand side. Furthermore, if $\lambda$ is not dominant integral for $G$ then the corresponding induced representation is 0 .

\section{A.5 Unitary irreducible representations of the conformal group}

Representations of the conformal group $S U(2,2)$ are usually specified by the quantum numbers $J_{1}, J_{2}$ the spins, and $L$ the dilation weight. These are related to the three Dynkin coefficients $n_{i}$ as follows:

$$
n_{1}=2 J_{1} \quad n_{2}=-L-J_{1}-J_{2} \quad n_{3}=2 J_{2} .
$$


Unitary irreducible highest weight representations satisfy the following unitary bounds [42] which we shall call a), b) and c) to tie in with the supersymmetric case:

$$
\begin{array}{rrlr}
\text { a) } J_{1} J_{2} \neq 0, \quad L \geq J_{1}+J_{2}+2 & \Rightarrow \quad n_{1} n_{3} \neq 0, \quad n_{1}+n_{2}+n_{3} \leq-2 \\
\text { b) } J_{1} J_{2}=0, \quad L \geq J_{1}+J_{2}+1 & \Rightarrow \quad n_{1} n_{3}=0, & n_{1}+n_{2}+n_{3} \leq-1 \\
\text { c) } J_{1}=J_{2}=0, L & =0 & \Rightarrow & n_{1}=n_{3}=0,
\end{array}
$$

Note that the central node $n_{2}$ is negative for all non-trivial representations, and thus according to the Borel-Weil theorem the representations are 0 if given as fields on complexified compactified Minkowski space $P \backslash S L(4)$ where $\mathrm{P}$ is given in (258). However we will be considering fields on affine Minkowski space which is an open subset of $P \backslash G$ and thus the Borel-Weil theorem no longer holds. This is because we are really considering representations of the non-compact group $S U(2,2)$. The negative node means that these representations must be infinite dimensional representations as all finite dimensional representations have dominant integral highest weights. The fact that we do not consider the whole of the space $P \backslash S L(4)$ also means that the induced representations are not always irreducible (see section 4 ).

\section{A.6 Projective twistor space}

It will be instructive to consider the twistor formalism, as we will be using similar techniques, although things are much simpler in the supersymmetric case. In twistor theory one considers a double fibration from complex Minkowski space. Projective Twistor space, $\mathbb{P T}$, is a subspace of $\mathbb{C P}^{3}$ which is the flag space $P \backslash S L(4)$, given by the following Dynkin diagram

$$
\mathbb{C P}^{3}=\longleftarrow
$$

There is a double fibration between $\mathbb{C P}^{3}$ and complex compactified Minkowski space which can be given in terms of Dynkin diagrams as

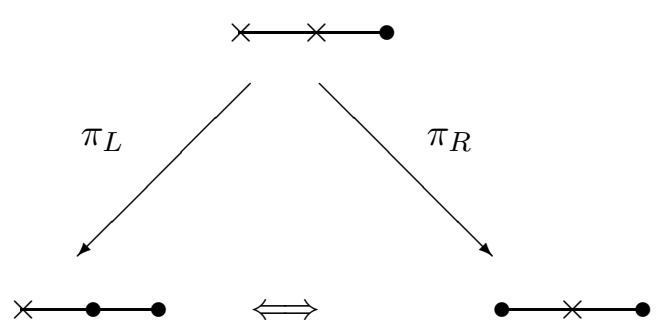

This induces a sub-double fibration from complex affine Minkowski space, $\mathbb{M}$ as follows

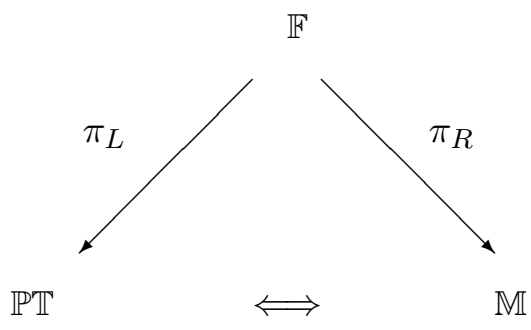


so that $\mathbb{F}=\pi_{R}^{-1} \mathbb{M}$ and $\mathbb{P} \mathbb{T}=\pi_{L} \mathbb{F}$. One then wishes to look at unitary irreducible representations of the conformal group. If we considered these representations as holomorphic fields on twistor space we would have the following Dynkin diagram

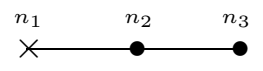

and since $n_{2}<0$ we do not have a finite dimensional representation of the parabolic subgroup. In twistor theory one gets around this by looking at higher cohomology. The result is that one can obtain the irreducible highest weight representations as higher cohomology classes on Twistor spaces without enforcing any constraints. In the supersymmetric case we do something similar, but things are much simpler as we do not have any negative nodes and therefore one does not have to consider higher cohomology classes. By looking at suitable spaces, one can obtain all unitary irreducible representations of the superconformal group as unconstrained holomorphic fields on these spaces.

\section{References}

[1] J. Maldacena, "The large N limit of superconformal field theories and supergravity," Adv. Theor. Math. Phys. 2 (1998) 231-252, hep-th/9711200.

[2] M. Flato and C. Fronsdal, "Representations of conformal supersymmetry," Lett. Math. Phys. 8 (1984) 159.

[3] V. K. Dobrev and V. B. Petkova, "All positive energy unitary irreducible representations of extended conformal supersymmetry," Phys. Lett. B162 (1985) 127.

[4] V. K. Dobrev and V. B. Petkova, "Group theoretical approach to extended conformal supersymmetry: function space realizations and invariant differential operators," Fortschr. Phys. 35 (1987) 537.

[5] B. Binegar, "Conformal superalgebras, massless representations and hidden symmetries," Phys. Rev. D34 (1986) 525.

[6] B. Morel, A. Sciarrino, and P. Sorba, "Unitary massless representations of conformal superalgebras,". Annecy Lab. Phys. Part. - LAPP-TH-142 (85,REC.OCT.) 10p.

[7] I. Bars and M. Gunaydin, "Unitary representations of noncompact supergroups," Commun. Math. Phys. 91 (1983) 31.

[8] M. Gunaydin and N. Marcus, "The spectrum of the $S^{5}$ compactification of the chiral $\mathrm{N}=2, \mathrm{D}=10$ supergravity and the unitary supermultiplets of $\mathrm{U}(2,2 / 4)$," Class. Quant. Grav. 2 (1985) L11.

[9] M. Gunaydin, D. Minic, and M. Zagermann, "4D doubleton conformal theories, CPT and II B string on AdS(5) x S(5)," Nucl. Phys. B534 (1998) 96-120, hep-th/9806042.

[10] M. Gunaydin, D. Minic, and M. Zagermann, "Novel supermultiplets of $\mathrm{SU}(2,2 \mid 4)$ and the AdS(5)/CFT(4) duality," Nucl. Phys. B544 (1999) 737, hep-th/9810226. 
[11] M. Gunaydin, "AdS/CFT dualities and the unitary representations of non- compact groups and supergroups: Wigner versus Dirac," hep-th/0005168.

[12] P. Heslop and P. Howe, "Harmonic superspaces and superconformal fields," hep-th/0009217.

[13] P. J. Heslop and P. S. Howe, "A note on composite operators in N = 4 SYM," hep-th/0106238.

[14] P. J. Heslop and P. S. Howe, "OPEs and 3-point correlators of protected operators in N = 4 SYM," hep-th/0107212.

[15] P. S. Howe and P. C. West, "Is N = 4 Yang-Mills theory soluble?," hep-th/9611074.

[16] P. S. Howe, E. Sokatchev, and P. C. West, "3-point functions in N = 4 Yang-Mills," Phys. Lett. B444 (1998) 341, hep-th/9808162.

[17] B. Eden, P. S. Howe, and P. C. West, "Nilpotent invariants in N = 4 SYM," Phys. Lett. B463 (1999) 19, hep-th/9905085.

[18] B. Eden, P. S. Howe, C. Schubert, E. Sokatchev, and P. C. West, "Extremal correlators in four-dimensional SCFT," Phys. Lett. B472 (2000) 323, hep-th/9910150.

[19] B. U. Eden, P. S. Howe, A. Pickering, E. Sokatchev, and P. C. West, "Four-point functions in N = 2 superconformal field theories," Nucl. Phys. B581 (2000) 523, hep-th/0001138.

[20] B. Eden, P. S. Howe, C. Schubert, E. Sokatchev, and P. C. West, "Four-point functions in $\mathrm{N}=4$ supersymmetric Yang-Mills theory at two loops," Nucl. Phys. B557 (1999) 355, hep-th/9811172.

[21] B. Eden, C. Schubert, and E. Sokatchev, "Three-loop four-point correlator in N =4 SYM," Phys. Lett. B482 (2000) 309, hep-th/0003096.

[22] A. Galperin, E. Ivanov, S. Kalitsyn, V. Ogievetsky, and E. Sokatchev, "Unconstrained $\mathrm{N}=2$ matter, Yang-Mills and supergravity theories in harmonic superspace," Class. Quant. Grav. 1 (1984) 469.

[23] A. Galperin, E. Ivanov, V. Ogievetsky, and E. Sokatchev, "Harmonic superspace: Key to $\mathrm{N}=2$ supersymmetry theories," JETP Lett. 40 (1984) 912-916.

[24] A. Galperin, E. Ivanov, S. Kalitsyn, V. Ogievetsky, and E. Sokatchev, "Unconstrained off-shell N=3 supersymmetric Yang-Mills theory," Class. Quant. Grav. 2 (1985) 155.

[25] A. S. Galperin, E. A. Ivanov, and V. I. Ogievetsky, "Superspaces for N=3 supersymmetry," Sov. J. Nucl. Phys. 46 (1987) 543.

[26] A. Galperin, E. Ivanov, V. Ogievetsky and E. Sokatchev, "Conformal Invariance In Harmonic Superspace," JINR-E2-85-363 In Batalin, I.A. (ed.) et al.: Quantum Field Theory and Quantum Statistics, vol. 2. Adam Hilger, Bristol, 1987, 233-248. 
[27] P. Heslop and P. S. Howe, "On harmonic superspaces and superconformal fields in four dimensions," Class. Quant. Grav. 17 (2000) 3743, hep-th/0005135.

[28] W. Siegel, "On-shell O(N) supergravity in superspace," Nucl. Phys. B177 (1981) 325.

[29] P. Howe, K. S. Stelle, and P. K. Townsend, "Supercurrents," Nucl. Phys. B192 (1981) 332 .

[30] P. S. Howe, K. S. Stelle, and P. K. Townsend, "Superactions," Nucl. Phys. B191 (1981) 445.

[31] P. S. Howe and G. G. Hartwell, "A Superspace survey," Class. Quant. Grav. 12 (1995) 1823.

[32] G. G. Hartwell and P. S. Howe, "(N, p, q) harmonic superspace," Int. J. Mod. Phys. A10 (1995) 3901-3920, hep-th/9412147.

[33] S. Ferrara and E. Sokatchev, "Superconformal interpretation of BPS states in AdS geometries," hep-th/0005151.

[34] J. Cornwell, Group Theory in Physics. Academic Press Inc., 1984.

[35] A. Ferber, "Supertwistors and conformal supersymmetry," Nucl. Phys. B132 (1978) 55.

[36] R. Baston and M. Eastwood, The Penrose Transform. Cambridge University Press, Cambridge, 1989.

[37] V. K. Dobrev, G. Mack, V. B. Petkova, S. G. Petrova, and I. T. Todorov 1977 "Harmonic analysis on the N-dimensional Lorentz group and its application to conformal quantum field theory," Lecture Notes in Physics 63 (Springer).

[38] V. K. Dobrev and V. B. Petkova, "Elementary representations and intertwining operators for the group SU*(4)," Rept. Math. Phys. 13 (1978) 233.

[39] V. K. Dobrev, "Elementary representations intertwining operators for $\mathrm{SU}(2,2)$. 1," J. Math. Phys. 26 (1985) 235.

[40] P. H. Dondi and P. D. Jarvis, "Diagram and superfield techniques in the classical superalgebras," J. Phys. A14 (1981) 547.

[41] S. Fernando, M. Gunaydin, and S. Takemae, "Supercoherent states of $\operatorname{OSp}\left(8^{*} \mid 2 \mathrm{~N}\right)$, conformal superfields and the $\operatorname{AdS}(7) / C F T(6)$ duality," hep-th/0106161.

[42] G. Mack, "All unitary ray representations of the conformal group SU(2,2) with positive energy," Commun. Math. Phys. 55 (1977) 1. 